\title{
Esboço geral e perspectivas da doença de Chagas no Nordeste do Brasil
}

\author{
General situation and perspectives of Chagas \\ disease in Northeastern Region, Brazil
}

João Carlos Pinto Dias 1

Evandro M. M. Machado 1

Antônia Lins Fernandes 2

Márcio C. Vinhaes 2

\footnotetext{
1 Centro de Pesquisas René Rachou, Fundação Oswaldo Cruz. C.P. 1743 , Belo Horizonte, $M G$ 30190-002, Brasil. jcpdias@cpqrr.fiocruz.br 2 Gerência Técnica de Doença de Chagas, Centro Nacional de Epidemiologia Fundação Nacional de Saúde, Ministério da Saúde.

Setor de Autarquias Sul, Brasília, $D F$ 70070-000, Brasil.
}

\begin{abstract}
Primary and secondary data show the importance and distribution of human Chagas disease (HCD) in Northeast Brazil. Among the 27 detected vector species, Triatoma infestans, Panstrongylus megistus, Triatoma brasiliensis and Triatoma pseudomaculata are epidemiologically important. Major medical impact is attributed to T. infestans and $\mathrm{P}$. megistus, the most domiciliated and vulnerable species, while the other two are native and more difficult to control. Regional differences in transmission and medical impact of HCD exist in the Northeast, where in general the disease appears to be less harmful than in other Brazilian regions like the Southeast and State of Goiás. There is a downward trend in HCD transmission and morbidity in the Northeast, its control in the region is a cause of concern because of the decommissioning of the National Health Foundation without a corresponding assimilation of its routine activities by regional and municipal institutions.
\end{abstract}

Key words Chagas Disease; Triatominae; Insect Vectors; Vector Control; Prevention and Control

Resumo Apresentam-se dados primários e secundários da doença de Chagas no Nordeste do Brasil, com prioridade para o vetor, a transmissão e o controle. Assinalam-se 27 espécies vetoras, com importância basicamente para Triatoma infestans, Panstrongylus megistus, Triatoma brasiliensis $e$ Triatoma pseudomaculata. As duas primeiras foram mais domiciliadas, causaram mais impacto médico-social e têm sido eliminadas mediante continuado controle químico. As duas últimas são nativas, ubiqüistas e mais peridomésticas, de difícil controle e menor impacto, remanescendo como grande desafio operacional. Há diversidade nos padrões de transmissão e da doença na região, explicáveis por diferentes situações ecológicas e sociais, possivelmente envolvendo diferentes cepas de Trypanosoma cruzi. A doença de Chagas é importante no Nordeste, embora com menor morbi-mortalidade aparente que no Sudeste e em Goiás. Há uma tendência à diminuição da transmissão e impacto da doença na região, mas preocupa a progressiva desativação regional da Fundação Nacional de Saúde, sem a correspondente absorção de suas atividades pelos Estados e municípios.

Palavras-chave Doença de Chagas; Triatominae; Insetos Vetores; Controle de Vetores; Prevenção e Controle 


\section{Introdução}

No contexto epidemiológico da doença de Chagas humana (DCH) no Brasil, a Região Nordeste (NE) ocupa importância acentuada, tendo sido a segunda em número de infectados e de índices de infestação triatomínica nos inquéritos nacionais de prevalência e distribuição dos vetores realizados entre 1975 e 1980 (Castro Filho \& Silveira, 1984; Fiusa-Lima \& Silveira, 1984; Silveira et al., 1984a). Passados vinte anos daquelas históricas observações, a região ainda preocupa em termos do risco de transmissão da DCH, lembrando-se, por exemplo, que, em 1996, o Programa de Controle da Doença de Chagas (PCDCh) da Fundação Nacional de Saúde/Ministério da Saúde (FNS/MS) capturou no Brasil 290.576 triatomíneos, sendo o Nordeste a região com maior número de capturas (201.156 exemplares), ou seja, 69,2\% do País. A preocupação leva em conta, particularmente, três situações: a) a região permanece socialmente muito deprimida e detentora dos mais altos índices de vivendas pobres apropriadas à colonização pelo triatomíneo no País; b) a região é o epicentro de dispersão de duas espécies de difícil controle pelos meios rotineiros da Fundação Nacional de Saúde, o Triatoma brasiliensis e o Triatoma pseudomaculata; e c) ocorrer hoje, nessa região, um baixo nível de cobertura operativa do programa de controle da doença de Chagas (PCDCh) pelas equipes da FNS, em razão do progressivo enxugamento dessa instituição em todo o país (Dias, 1998)

De modo geral, a elaboração do presente suplemento dos Cadernos de Saúde Pública procurou abordar a doença de Chagas no Nordeste do Brasil com vistas a fazer uma revisão da situação epidemiológica e apontar algumas perspectivas de encaminhamento e análise. No bojo da Iniciativa do Cone Sul, toda centrada na eliminação do Triatoma infestans e da doença de Chagas transfusional, a situação do nordeste brasileiro é bastante especial, em função da presença de outras espécies vetoras do Trypanosoma cruzi e das características sociais, políticas e econômicas da área, geralmente entendidas como complicadoras ao sucesso do programa. Em particular, o presente texto acompanha as informações gerais para o país (ver Vinhaes \& Dias, neste fascículo) e discrimina e amplia algumas situações e problemas específicos do Nordeste. Em sua preparação, buscou-se sintetizar as informações esparsas ou pontuais que os gerentes regionais da FNS levaram à discussão no seminário de Praia das Fontes, Ceará, em 1998, em cotejo com os dados de rotina repassados à Gerência Técnica de
Chagas da FNS/Brasília, envolvendo, ainda, a experiência direta dos autores na área. Dado o caráter desta publicação, o enfoque central foi pertinente ao controle da transmissão vetorial e ao programa antitriatomínico levado a cabo na área entre 1980 e 1999. Não obstante, dados e observações sobre a transmissão transfusional e algumas questões de morbi-mortalidade foram apensadas ao texto para uma melhor avaliação do peso médico e social da doença de Chagas nessa importante e preocupante região brasileira.

\section{Material e métodos}

O Nordeste é uma região importante no Brasil, apresentando, no censo de 1991, uma população total de 42.497 .540 habitantes $(28,94 \%$ da população brasileira). Tem uma área 1.561.178 $\mathrm{km}^{2}$ (18,27\% da área do país) e corresponde a nove estados, fazendo limite com as Regiões Norte, Sudeste e Centro-Oeste do Brasil (Figura 1). A Tabela 1 explicita a área e a população por Unidade da Federação (UF), com as respectivas densidades populacionais. De modo geral, o crescimento da população nordestina tem sido menor que o da população brasileira como um todo, representando 35\% dela em 1940, 30,18\% em 1970 e 28, 50\% em 1996 (Tabelas 1 e 2). É também uma região de pequenas densidades populacionais, especialmente nas áreas rurais, apresentando, em 1996, uma densidade regional de $27,2 \mathrm{hab} / \mathrm{km}^{2}$, maior que a do Brasil, de modo geral $\left(18,49 \mathrm{hab} / \mathrm{km}^{2}\right.$, incluída a Amazônia); Piauí e Maranhão mostram as menores densidades populacionais da região $(10,2$ $\mathrm{hab} / \mathrm{km}^{2}$ e $14,8 \mathrm{hab} / \mathrm{km}^{2}$ ), sendo mais densos Alagoas e Sergipe (Tabela 1).

O Nordeste é também uma das regiões mais pobres do País, ainda muito ruralizada e apresentando, no Brasil, os maiores índices de habitações humanas de baixa qualidade e muito próprias como abrigo de triatomíneos. Em paralelo, é uma região que historicamente apresenta os mais altos níveis de emigração do Brasil, especialmente em direção ao Sudeste e ao Norte (Coura, 1984; Alencar, 1987). Na Tabela 2, observam-se as evoluções da população total e rural do Nordeste como um todo e por estado, verificando-se que naquela região o processo de crescimento populacional e os índices de urbanização foram progressivos desde 1940. Nota-se que um terço da população nordestina ainda vivia em zona rural em 1996, sendo maiores as taxas para o Maranhão e o Piauí, e a menor em Pernambuco (essa última ainda significativamente maior que a do País como um todo). 
Quanto à endemia chagásica, no inquérito nacional de 1978-1980, o Nordeste apresentou uma prevalência geral de 3,05\% da infecção chagásica (Brasil = 4,40\%), com índices significativos de DCH nos Estados da Bahia, Sergipe, Alagoas e Piauí (Fiusa-Lima \& Silveira, 1984). Para o atual trabalho, basicamente, compulsaram-se os dados secundários disponíveis na Gerência Técnica de Doença de Chagas, do Centro Nacional de Epidemiologia/Fundação Nacional de Saúde - CENEPI/FNS, em Brasília, seguindo um roteiro de avaliação atual (19971999) e prévio (linha de base em 1980-1982, quando o PCDCh foi priorizado no Ministério da Saúde). O enfoque básico foi, naturalmente, o conjunto de dados entomológicos e de controle, sintetizados em tabelas comparativas por estado e por época. Igualmente, dados oficiais da Coordenação de Sangue e Hemoderivados do Ministério da Saúde (COSAH) foram incorporados à análise. Dados disponíveis ou pesquisados sobre a transmissão da doença e seu impacto de morbi-mortalidade foram acrescentados ao texto, para relativizar o panorama entomológico. A metodologia de pesquisa e os indicadores triatomínico-tripanossômicos e operacionais utilizados são aqueles tradicionais da FNS, referendados pela Organização Mundial de Saúde (OMS) (SUCAM, 1980; WHO, 1991). A determinação seletiva da importância das espécies foi determinada conforme os critérios de Silveira et al. (1984b). Complementarmente, supervisões e visitas de campo efetuadas em 1999 por dois dos autores foram de muita ajuda para verificar dados locais e colher impressões diretas sobre o desempenho do PCDCh e a evolução da endemia chagásica na área. Uma série de trabalhos pioneiros sobre a doença de Chagas no Nordeste foram consultados, sendo os principais referidos na bibliografia deste texto, o mesmo ocorrendo para alguns aspectos clínicos e de morbi-mortalidade. Com o intuito de homogeneizar-se o material, sempre que possível, foram trabalhados os dados por estado, em tabelas comparativas com a situação do Brasil e de suas macrorregiões, utilizando-se os índices preconizados pela Organização Mundial da Saúde (WHO, 1991).

\section{Antecedentes mais remotos}

A primeira referência à existência da endemia no Nordeste deve-se a Carlos Chagas, numa entrevista em 1911, quando a relata "no sul da Bahia”, além de presente em Minas Gerais, Goiás, Mato Grosso e, talvez, São Paulo (A Imprensa, 1911). A seguir, a DCH foi detectada no Nordes-

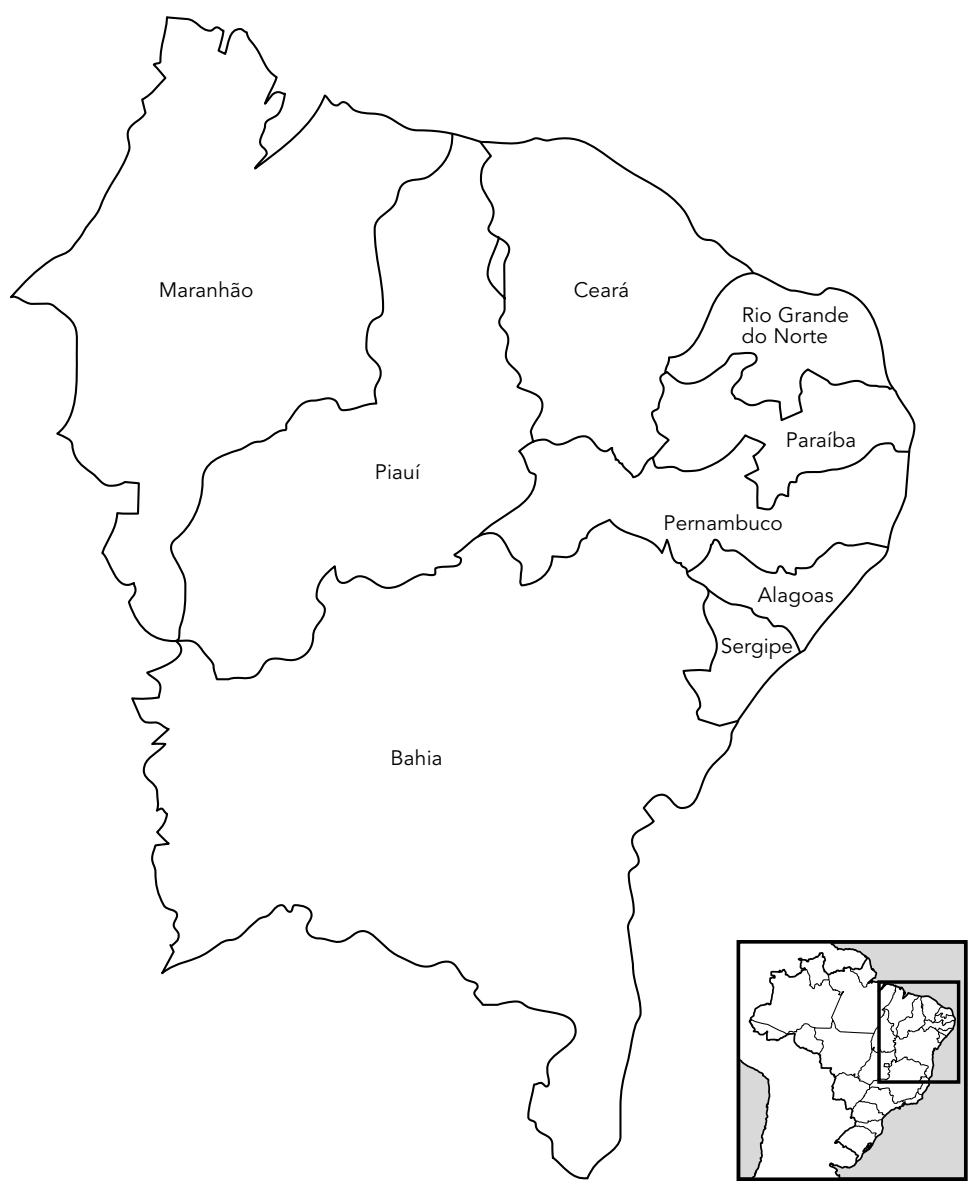

te, especialmente a partir do relato de triatomíneos domiciliados em vastas extensões da Bahia, de Pernambuco, de Sergipe e do Ceará (Pirajá-da-Silva, 1913; Lutz \& Machado, 1915; Lucena, 1952; Alencar, 1987; Carcavallo et al., 1997; Dias \& Schofield, 1998, entre outros).

Em 1911, Arthur Neiva (Neiva, 1911) descreveu o Triatoma brasiliensis, espécie nova do Ceará, referindo-se que daquele estado, em 1859 e 1870, Stal recebera e descrevera Rhodnius nasutus. Não obstante, certamente, a doença humana já ocorria endemicamente na região há pelo menos um século antes de Chagas, a julgar por evidências clínicas e históricas. O marcador mais concreto da presença da infecção humana em várias áreas é o megaesôfago endêmico, afecção prevalente em vastas áreas brasileiras onde ocorre a transmissão da esqui- 
Tabela 1

Área, população e densidade populacional do Nordeste brasileiro

e dos estados da região em 1991.

\begin{tabular}{lrrc}
\hline & Área absoluta (km²) & População & Densidade (hab/km²) \\
\hline Nordeste & $1.561 .177,8$ & 42.497 .540 & 27,2 \\
Alagoas & $27.933,1$ & 2.514 .100 & 90,0 \\
Bahia & $567.295,3$ & 11.867 .991 & 20,9 \\
Ceará & $146.348,3$ & 6.366 .647 & 43,5 \\
Maranhão & $333.365,6$ & 4.930 .253 & 14,8 \\
Paraíba & $56.584,6$ & 3.201 .114 & 48,7 \\
Pernambuco & $98.937,8$ & 7.127 .855 & 72,0 \\
Piauí & $252.378,5$ & 2.582 .137 & 10,2 \\
Rio Grande do Norte & $53.306,8$ & 2.415 .567 & 45,3 \\
Sergipe & $22.050,4$ & 1.491 .876 & 67,7 \\
Regiões em litígio & $2.947,4$ & - & - \\
\hline
\end{tabular}

Fonte: IBGE, 1991.
Piauí, em 1982; b) O modelo de controle da DCH transfusional e de outras moléstias veiculadas por hemoterapia foi gestado e implantado de modo pioneiro em Pernambuco, pelo HEMOPE - Hemocentro de Pernambuco (Dr. Luís Gonzaga dos Santos) na década de 60; c) Dentre os primeiros serviços regulares de atenção médica ao chagásico, sobressaiu-se o modelar ambulatório do Hospital Oswaldo Cruz, em Recife (Dr. Wilson Oliveira Jr. e colaboradores), em 1987.

Também, muito particular e notório é o fato de que o Triatoma infestans, introduzido na região por transporte passivo após 1950, dispersou-se rapidamente por vários Estados até, aproximadamente, 1983, sendo registrado em todos os Estados nordestinos, menos no Ceará e no Rio Grande do Norte. Foi, então, duramente combatido pela SUCAM/FNS (Superintendência de Campanhas de Saúde Pública/Fundação Nacional de Saúde), combate esse definitivamente priorizado pela Iniciativa do Cone Sul, em 1992.

Com isso, hoje houve uma drástica redução zotripanose. A etiologia chagásica desse agravo, bastante conhecido no Brasil desde o século passado, só foi suspeitada alguns anos depois da descoberta de Chagas, por Ulysses $\mathrm{Pa}$ ranhos, em 1913, e pelo próprio Carlos Chagas (Chagas, 1916), sendo a comprovação inequívoca somente realizada quatro décadas mais tarde (Rezende, 1998).

Na sua revisão, Rezende (1998) aponta referências feitas por Spix e Martius, entre 1820 e 1823, ao "mal de engasgo", endêmico na região de Oeiras, Piauí. Também uma expedição de Neiva e Machado (Neiva \& Pena, 1916) assinala e descreve centenas de casos de mal de engasgo na Bahia, Pernambuco e Goiás, também reportando, nessas regiões, casos de "avexame", que Chagas, Dias e outros atribuíram à cardiopatia esquizotripanósica (Chagas, 1930; Dias et al., 1945). No Ceará, em 1921, Gavião Gonzaga verificou triatomíneos infectados no Cariri e em Quixadá, sendo diagnosticados os primeiros casos humanos, por xenodiagnóstico, somente em 1942 (Alencar, 1987).

Muitos destaques históricos pertinentes à doença de Chagas no Nordeste, especialmente a partir dos anos 50, advêm de pesquisas sucessivas e importantes de eminentes cientistas como Durval Lucena, Ruy João Marques, Joaquim Eduardo Alencar, Emmanuel Dias, J. Rodrigues Coura, Aluísio Prata, Vanise Macedo, Zilton e Sônia Andrade, Ítalo Scherlock e outros. Assinalam-se, ainda, três fatos muito relevantes da história contemporânea da DCH e de seu controle, marcados pelo Nordeste do Brasil: a) O programa atual de controle da DCH no Brasil foi inaugurado pelo Ministro Waldir Arcoverde, no dos focos, com eliminação da espécie em vários municípios e, mesmo, estados (Maranhão, Paraíba, Pernambuco, Sergipe, Alagoas e Piauí), permanecendo ainda hoje um foco significativo na Bahia.

Finalmente, em termos do controle do triatomismo domiciliário, o Nordeste é, no País, a região endêmica com maiores problemas presentes e futuros, por ser o centro de dispersão e maior concentração de Triatoma brasiliensis, o "barbeiro" atualmente mais preocupante e mais difícil de controlar em todo o País (Alencar, 1987; Dias \& Coura, 1997; Silveira \& Vinhaes, 1998).

\section{Resultados}

\section{Entomologia e controle}

Já foram identificadas no Nordeste do Brasil cerca de 27 espécies ou subespécies de triatomíneos transmissores do Trypanosoma cruzi, correspondendo a mais da metade daquelas detectadas no Brasil (Carcavallo et al., 1997). Na Tabela 3, discriminam-se as espécies por Unidade da Federação do NE, verificando-se que há grande diversidade de gêneros e espécies em várias UFs e que a dispersão por espécie também varia.

Chama a atenção, especialmente, a grande dispersão de Panstrongylus megistus, T. brasiliensis, T. pseudomaculata e, mesmo, T. infestans. O T. brasiliensis e o T. pseudomaculata são espécies nativas, com epicentro de dispersão no Nordeste (Forattini, 1980). O gênero Rhodnius se espraia mais pelo norte da região, sendo $R$. 
Evolução da população do Brasil e dos estados do Nordeste brasileiro entre 1940 e 1996, com a proporção de residentes em área rural.

\begin{tabular}{|c|c|c|c|c|c|c|c|c|c|c|c|}
\hline \multirow{2}{*}{$\begin{array}{l}\text { População } \\
\text { residente }\end{array}$} & \multirow[b]{2}{*}{ Brasil } & \multirow[b]{2}{*}{ Nordeste } & \multirow[b]{2}{*}{ MA } & \multirow[b]{2}{*}{$\mathrm{PI}$} & \multicolumn{4}{|c|}{ Grandes regiões e Unidades Federativas } & \multirow[b]{2}{*}{$A L$} & \multirow[b]{2}{*}{ SE } & \multirow[b]{2}{*}{ BA } \\
\hline & & & & & CE & RN & PB & PE & & & \\
\hline \multicolumn{12}{|l|}{1940} \\
\hline total & 41.236 .315 & 14.434 .080 & 1.235 .169 & 817.601 & 2.091 .032 & 768.018 & 1.422 .282 & 2.688 .240 & 951.360 & 542.326 & 3.918 .112 \\
\hline$\%$ rural & 68,76 & 76,58 & 84,98 & 84,81 & 77,28 & 78,61 & 78,11 & 70,69 & 75,91 & 69,35 & 76,07 \\
\hline \multicolumn{12}{|l|}{1950} \\
\hline total & 51.944 .397 & 17.973 .413 & 1.583 .248 & 1.045 .696 & 2.695 .450 & 967.921 & 1.713 .259 & 3.395 .185 & 1.093 .137 & 644.361 & 4.834 .575 \\
\hline$\%$ rural & 63,84 & 73,60 & 82,68 & 83,69 & 74,79 & 73,78 & 73,34 & 65,61 & 73,80 & 68,19 & 74,13 \\
\hline \multicolumn{12}{|l|}{1960} \\
\hline total & 70.070 .547 & 22.181 .880 & 2.469 .447 & 1.242 .136 & 3.296 .366 & 1.145 .502 & 2.000 .851 & 4.095 .379 & 1.258 .107 & 752.256 & 5.920 .447 \\
\hline$\%$ rural & 55,33 & 66,11 & 82,32 & 77,01 & 66,66 & 62,63 & 65,15 & 55,43 & 66,58 & 61,46 & 65,66 \\
\hline \multicolumn{12}{|l|}{1970} \\
\hline total & 93.139 .037 & 28.111 .927 & 2.992 .686 & 1.680 .573 & 4.361 .603 & 1.550 .244 & 2.382 .617 & 5.160 .640 & 1.588 .109 & 900.744 & 7.493 .470 \\
\hline$\%$ rural & 44,08 & 58,19 & 74,87 & 68,07 & 58,19 & 52,44 & 57,94 & 45,53 & 60,22 & 53,88 & 58,83 \\
\hline \multicolumn{12}{|l|}{1980} \\
\hline total & 119.002 .706 & 38.812 .356 & 3.996 .404 & 2.139 .021 & 5.288 .253 & 1.898 .172 & 2.770 .176 & 6.141 .993 & 1.982 .591 & 1.140 .121 & 9.454 .346 \\
\hline$\%$ rural & 32,41 & 49,54 & 68,59 & 58,02 & 46,86 & 41,25 & 47,69 & 38,40 & 50,74 & 45,81 & 50,70 \\
\hline \multicolumn{12}{|l|}{1991} \\
\hline total & 146.825 .475 & 42.947 .540 & 4.930 .253 & 2.582 .137 & 6.366 .647 & 2.415 .567 & 3.201 .114 & 7.127 .855 & 2.514 .100 & 1.491 .876 & 11.867 .991 \\
\hline$\%$ rural & 24,41 & 39,35 & 59,99 & 47,05 & 34,63 & 30,89 & 35,89 & 29,13 & 41,05 & 32,78 & 40,88 \\
\hline \multicolumn{12}{|l|}{1996} \\
\hline total & 157.070 .163 & 44.766 .851 & 5.222 .183 & 2.673 .085 & 6.809 .290 & 2.558 .660 & 3.305 .616 & 7.399 .071 & 2.633 .251 & 1.624 .020 & 12.541 .675 \\
\hline$\%$ rural & 21,64 & 34,79 & 48,08 & 41,78 & 30,78 & 27,95 & 31,57 & 25,98 & 36,89 & 29,78 & 37,59 \\
\hline
\end{tabular}

Fonte: IBGE, 1999.

nasutus o mais disperso e Rhodnius neglectus o mais meridional. Rhodnius pictipes, Rhodnius robustus e Rhodnius brethesi são as espécies mais setentrionais na região, sendo o Maranhão o estado nordestino com mais espécies desse gênero, certamente, por sua condição de proximidade com a Amazônia, onde abundam os Rhodnius (Schofield, 1994; Carcavallo et al., 1997). Nenhuma espécie desse gênero é exclusiva da parte sul do Nordeste. Ao contrário, ao norte, são exclusivos os achados de $R$. brethesi, $R$. pictipes e R. robustus. A Bahia, o estado nordestino mais meridional, é o que apresenta maior variedade de espécies, concentrando particularmente os gêneros Triatoma e Panstrongylus, enquanto que o Maranhão, no extremo norte da região, é o segundo estado em número de espécies detectadas. Na mesma linha, a Bahia parece ser o limite setentrional de espécies como Panstrongylus diasi, Triatoma len- ti e Triatoma vitticeps, talvez, Cavernicola pilosa e Parabelminus yurupucu, enquanto Eratyrus mucronatus e Panstrongylus lignarius só foram detectados no Maranhão (Tabela 3). Há que se notar, ainda, que existem referências isoladas de achados de mais duas espécies, além daquelas listadas na Tabela 3: o Rhodnius prolixus foi assinalado no Ceará (Neiva, 1911; Pinto, 1925) e o Triatoma rubrovaria na Bahia (Pinto, 1925; Neiva \& Lent, 1941). Ambas não constam na Tabela 3 por tratarem-se de menções muito esporádicas e por ter, particularmente, o R. prolixus, na época, restrições à sua classificação, segundo o próprio Neiva (1911). Das 11 espécies registradas do gênero Triatoma no Nordeste, todas são assinaladas ao sul da região (Bahia), onde o gênero predomina, sendo que nenhuma é exclusiva do norte nordestino. O T. brasiliensis e o T. pseudomaculata se dispersam a partir do Nordeste e têm sido detec- 
Registro de triatomíneos nos estados do Nordeste brasileiro, entre 1912 e 1999, segundo o Ministério da Saúde e diversos autores.

\begin{tabular}{|c|c|c|c|c|c|c|c|c|c|c|c|c|}
\hline \multirow[t]{2}{*}{ Espécie } & \multirow[b]{2}{*}{ BA } & \multirow[b]{2}{*}{ SE } & \multirow[b]{2}{*}{$A L$} & \multicolumn{3}{|c|}{ Estados } & \multirow[b]{2}{*}{ CE } & \multirow[b]{2}{*}{$\mathrm{PI}$} & \multirow[b]{2}{*}{ MA } & \multicolumn{2}{|c|}{$\begin{array}{l}\text { Presença da } \\
\text { espécie nos } \\
\text { estados }\end{array}$} & \multirow[t]{2}{*}{ Observação } \\
\hline & & & & PE & PB & $\mathrm{RN}$ & & & & $\mathrm{n}$ & $\%$ & \\
\hline Cavernicola pilosa & $x$ & & & & & & & & & 1 & 11,1 & - \\
\hline Eratyrus mucronatus & & & & & & & & & $x$ & 1 & 11,1 & - \\
\hline Panstrongylus diasi & $x$ & & & & & $x$ & & & & 2 & 22,2 & - \\
\hline Panstrongylus geniculatus & $x$ & & & & & & $x$ & & $x$ & 3 & 33,3 & - \\
\hline Panstrongylus lignarius & & & & & & & & & $x$ & 1 & 11,1 & - \\
\hline Panstrongylus lutzi & $x$ & $x$ & $x$ & $x$ & $x$ & $x$ & $x$ & $x$ & & 8 & 88,9 & - \\
\hline Panstrongylus megistus & $x$ & $x$ & $x$ & $x$ & $x$ & $x$ & $x$ & $x$ & $x$ & 9 & 100,0 & - \\
\hline Parabelminus yurupucu & $x$ & & & & & & & & & 1 & 11,1 & - \\
\hline Psammolestes tertius & $x$ & & & $x$ & $x$ & & $x$ & & & 4 & 44,4 & - \\
\hline Rhodnius brethesi & & & & & & & & $x$ & $x$ & 2 & 22,2 & - \\
\hline Rhodnius domesticus & $x$ & & & & & & & $x$ & $x$ & 3 & 33,3 & - \\
\hline Rhodnius nasutus & & & & $x$ & $x$ & $x$ & $x$ & $x$ & $x$ & 6 & 66,7 & - \\
\hline Rhodnius neglectus & $x$ & $x$ & & $x$ & & & & $x$ & $x$ & 5 & 55,5 & - \\
\hline Rhodnius pictipes & & & & & & & & $x$ & $x$ & 2 & 22,2 & - \\
\hline Rhodnius robustus & & & & & & & & & $x$ & 1 & 11,1 & - \\
\hline Triatoma arthurneivai & $x$ & & & & & & & $x$ & & 2 & 22,2 & - \\
\hline Triatoma brasiliensis & $x$ & $x$ & $x$ & $x$ & $x$ & $x$ & $x$ & $x$ & $x$ & 9 & 100,0 & NE é seu epicentro de dispersão \\
\hline Triatoma costalimai & $x$ & & & & & & & & & 1 & 11,1 & - \\
\hline Triatoma infestans & $x$ & $x$ & $x$ & $x$ & $x$ & & & $x$ & $x$ & 7 & 77,8 & $\begin{array}{l}\text { Introduzido nos anos } 50 \text { e em } \\
\text { desaparecimento em } \mathrm{AL}, \mathrm{PB}, \mathrm{SE} \text {, } \\
\mathrm{MA} \text { e } \mathrm{Pl} \text {, entre } 1990 \text { e } 1998\end{array}$ \\
\hline Triatoma lenti & $x$ & & & & & & & & & 1 & 11,1 & $\begin{array}{l}\text { Inclui capturas assinaladas como } \\
\text { Triatoma pessoai }\end{array}$ \\
\hline Triatoma melanocephala & $x$ & $x$ & & $x$ & $x$ & $x$ & & & & 5 & 55,5 & - \\
\hline Triatoma petrochiae & $x$ & & & $x$ & $x$ & $x$ & & & & 4 & 44,4 & - \\
\hline Triatoma pseudomaculata & $x$ & $x$ & $x$ & $x$ & $x$ & $x$ & $x$ & $x$ & $x$ & 9 & 100,0 & NE é seu epicentro de dispersão \\
\hline Triatoma rubrofasciata & $x$ & $x$ & $x$ & $x$ & $x$ & $x$ & & & $x$ & 7 & 77,8 & Focos portuários (marítimos) \\
\hline Triatoma sordida & $x$ & & & $x$ & & & & $x$ & $x$ & 4 & 44,4 & - \\
\hline Triatoma tibiamaculata & $x$ & $x$ & $x$ & $x$ & & & & & & 4 & 44,4 & - \\
\hline Triatoma vitticeps & $x$ & & & & & & & & & 1 & 11,1 & Somente divisa com Minas Gerais \\
\hline Número de espécies/estado & 21 & 9 & 7 & 13 & 10 & 9 & 7 & 12 & 15 & & & Total de espécies $=27$ \\
\hline$\%$ de espécies/estado & 77,8 & 33,3 & 25,9 & 48,2 & 37,0 & 33,3 & 25,9 & 44,4 & 55,6 & & & \\
\hline
\end{tabular}

tados em todos os estados. As duas se constituem na maior preocupação do PCDCh na região, por sua dispersão e dificuldades ao controle. No seu conjunto, o quadro conforma uma imagem em espelho para o gênero Rhodnius, predominante setentrionalmente no Nordeste, e Triatoma, ao sul da região (Tabela 3). Do gênero Panstrongylus são detectadas espécies ao norte e ao sul, estando mais dispersos Panstrongylus megistus e Panstrongylus lutzi.

Em termos de importância para a saúde pública, considerando-se seletivamente as taxas de dispersão, infestação predial, colonização do intradomicílio, infecção natural pelo T. cru$z i$, antropofilia e número total de capturas, as espécies triatomínicas responsáveis pela ocorrência da DCH no Nordeste têm sido basicamente $T$. brasiliensis, $P$. megistus, $T$. infestans, $T$. pseudomaculata e ainda, provavelmente, $R$. nasutus e T. sordida (Neiva \& Lent, 1941; Castro Filho \& Silveira, 1984; Silveira et al., 1984a; Alencar, 1987). Discriminando-se cronologicamente, apresentam-se três cenários históricos do triatomismo no NE, a saber: 1) até a década 50 , antes da chegada de $T$. infestans à região, é provável que o grosso da transmissão da $\mathrm{DCH}$ 
no Nordeste ocorreu basicamente por meio de P. megistus e T. brasiliensis, em suas áreas próprias de ocorrência; 2 ) entre a década de 50 e fim da década de 80, T. infestans invadiu a região e certamente somou-se às anteriores em importância, especialmente nos Estados da Bahia, Pernambuco, Paraíba e sul do Piauí; 3) a partir de 1990, T. infestans perde a importância em toda a região, remanescendo basicamente um foco relevante, na Bahia, à margem esquerda do Rio São Francisco. Também P. megistus diminui suas densidades em praticamente todo o Nordeste, restando como espécie mais importante o T. brasiliensis. Ao longo dos últimos lustros, lenta e progressivamente, T. pseudomaculata vem se instalando nos ecótopos artificiais, alcançando algum grau de antropofilia e aumentando suas taxas de colonização em vários estados, tornando-se, assim, uma espécie em relativa ascensão, merecedora de estudo e acompanhamento. As demais espécies assinaladas, todas encontráveis em ecótopos naturais, não têm apresentado tendência forte à domiciliação, nos últimos vinte ou trinta anos, carecendo até aqui de importância epidemiológica quanto à transmissão da DCH. A Tabela 4 explicita parte dessa história, mostrando a freqüência relativa das principais espécies (incluindo T. sordida) entre 1977 e 1997, complementando-se para a década atual as ocorrências entre 1992 e 1997, na Tabela 5.

De modo geral, observa-se que realmente foram T. brasiliensis e P. megistus as espécies mais presentes no contexto do passado, ressal-

\section{Tabela 4}

Freqüência relativa (\%) das principais espécies de triatomíneos capturados pela SUCAM/FNS nos estados do Nordeste brasileiro em 1977 e 1997.

\begin{tabular}{|c|c|c|c|c|c|c|c|c|c|c|c|c|c|c|c|c|c|c|}
\hline \multirow[t]{3}{*}{ Espécie } & \multicolumn{18}{|c|}{ Estado } \\
\hline & \multicolumn{2}{|c|}{$\mathrm{AL}$} & \multicolumn{2}{|c|}{ BA } & \multicolumn{2}{|c|}{ CE } & \multicolumn{2}{|c|}{ MA } & \multicolumn{2}{|c|}{ PB } & \multicolumn{2}{|c|}{ PE } & \multicolumn{2}{|c|}{ PI } & \multicolumn{2}{|c|}{$\mathrm{RN}$} & \multicolumn{2}{|c|}{ SE } \\
\hline & 1977 & 1997 & 1977 & 1997 & 1977 & 1997 & 1977 & 1997 & 1977 & 1997 & 1977 & 1997 & 1977 & 1997 & 1977 & 1997 & 1977 & 1997 \\
\hline T. brasiliensis & 0,0 & 15,0 & 10,0 & 10,0 & 51,0 & 56,0 & $\mathrm{NI}$ & 4,0 & 13,0 & 62,0 & 5,0 & 33,0 & 80,0 & 62,0 & 26,0 & 43,0 & 0,0 & 0,0 \\
\hline T. pseudomaculata & 0,0 & 69,0 & 18,0 & 21,5 & 30,0 & 42,0 & $\mathrm{NI}$ & 6,0 & 51,0 & 28,0 & 25,0 & 47,0 & 15,0 & 35,0 & 69,0 & 39,0 & 64,0 & 100,0 \\
\hline P. megistus & 100,0 & 11,0 & 36,0 & 0,5 & 16,0 & 0,5 & $\mathrm{NI}$ & 0,5 & 33,0 & 0,3 & 65,0 & 8,0 & 2,0 & 0,4 & 0,0 & 0,1 & 36,0 & 0,0 \\
\hline T. infestans & 0,0 & 0,0 & 18,0 & 1,0 & 0,0 & 0,0 & $\mathrm{NI}$ & 0,0 & 0,0 & 0,0 & 0,0 & 0,1 & 0,0 & 0,1 & 0,0 & 0,0 & 0,0 & 0,0 \\
\hline T. sordida & 0,0 & 0,0 & 18,0 & 66,5 & 0,0 & 0,0 & $\mathrm{NI}$ & 0,1 & 0,0 & 0,0 & 0,0 & 0,0 & 0,0 & 2,0 & 0,0 & 0,0 & 0,0 & 0,0 \\
\hline Outras & 0,0 & 5,0 & 0,0 & 0,5 & 3,0 & 1,5 & $\mathrm{NI}$ & $89,4^{*}$ & 3,0 & 9,7 & 5,0 & 11,9 & 3,0 & 0,5 & 5,0 & 17,9 & 0,0 & 0,0 \\
\hline
\end{tabular}

* Triatoma rubrofasciata $=73,3 \%$

$\mathrm{NI}=$ não informado.

Fontes: Castro Filho \& Silveira, 1979 e Silveira \& Vinhaes, 1998.

Espécie numericamente predominante dos triatomíneos capturados nos domicílios por Unidade Federativa pela Fundação Nacional de Saúde no Nordeste brasileiro entre 1992 e 1997.

\begin{tabular}{|c|c|c|c|c|c|}
\hline \multirow[t]{2}{*}{ Unidade Federativa } & \multicolumn{5}{|c|}{ Espécie predominante } \\
\hline & 1992 & 1993 & 1994 & 1995 & 1997 \\
\hline Alagoas & T. pseudomaculata & P. megistus & P. megistus & P. megistus & T. pseudomaculata \\
\hline Bahia & T. sordida & T. sordida & T. sordida & T. sordida & T. sordida \\
\hline Ceará & T. brasiliensis & T. brasiliensis & T. brasiliensis & T. pseudomaculata & T. brasiliensis \\
\hline Maranhão & T. rubrofasciata & T. rubrofasciata & T. rubrofasciata & T. rubrofasciata & T. rubrofasciata \\
\hline Paraíba & T. pseudomaculata & T. pseudomaculata & T. brasiliensis & T. pseudomaculata & T. brasiliensis \\
\hline Pernambuco & P. megistus & P. megistus & T. brasiliensis & T. brasiliensis & T. pseudomaculata \\
\hline Piauí & T. pseudomaculata & T. brasiliensis & T. brasiliensis & T. brasiliensis & T. brasiliensis \\
\hline Rio Grande do Norte & T. pseudomaculata & T. pseudomaculata & T. brasiliensis & T. pseudomaculata & T. brasiliensis \\
\hline Sergipe & T. pseudomaculata & T. pseudomaculata & T. pseudomaculata & T. pseudomaculata & T. pseudomaculata \\
\hline
\end{tabular}


tando-se a progressiva domiciliação de T. pseudomaculata em vários estados, mormente em Pernambuco e Ceará. T. infestans apresenta uma história particular de introdução regional, sendo espécie dominante apenas na Bahia, durante algum tempo, estando, hoje, em vias de eliminação. Os dados da FNS mostram que ao final dos anos 80 havia sido assinalada a espécie em 120 municípios de quatro Estados nordestinos, sendo 95 na Bahia, 15 em Pernambuco, seis no Piauí e quatro na Paraíba. Com sua priorização no PCDCh, já em 1994, estava restrita a 48 municípios (60\% de redução) e, em 1996, restavam 25 municípios infestados $(79,2 \%$ de redução), correspondendo a vinte municípios na Bahia, três em Pernambuco e dois no Piauí. Em 1999, ampla revisão demonstrou a eliminação da espécie também em Pernambuco e no Piauí, segundo as regionais da FNS.

Para P. megistus, em termos gerais, os dados recentes mostram uma grande redução em todas as áreas investigadas, remanescendo apenas focos em Pernambuco e Alagoas (onde dominava), correspondendo apenas a $1 \%$ dos triatomíneos capturados no Nordeste em 1997 (Tabela 6). Acha-se virtualmente eliminado de Ser-

Principais espécies de triatomíneos capturados por estado do Nordeste brasileiro e sua proporção no estado e na região, em 1998.

\begin{tabular}{|c|c|c|c|c|c|c|c|c|c|c|}
\hline & $A L$ & BA & CE & MA & PB & PE & PI & RN & SE & Nordeste \\
\hline $\begin{array}{l}\text { Número de unidades } \\
\text { pesquisadas }\end{array}$ & 14.088 & 246.286 & 282.050 & 30.583 & 50.476 & 55.119 & 89.169 & 483 & 6.949 & 775.203 \\
\hline \multicolumn{11}{|l|}{$\begin{array}{l}\text { Número de triatomíneos } \\
\text { capturados por espécie, } \\
\text { por estado, no Nordeste } \\
\text { do Brasil - } 1997\end{array}$} \\
\hline \multicolumn{11}{|l|}{ Triatoma infestans } \\
\hline $\mathrm{n}$ & 0 & 585 & 0 & 0 & 0 & 7 & 14 & 0 & 0 & 606 \\
\hline$\% *$ & 0,00 & 1,21 & 0,00 & 0,00 & 0,00 & 0,08 & 0,11 & 0,00 & 0,00 & 0,43 \\
\hline \multicolumn{11}{|l|}{ Panstrongylus megistus } \\
\hline $\mathrm{n}$ & 62 & 281 & 323 & 2 & 17 & 693 & 47 & 3 & 0 & 1.428 \\
\hline$\% *$ & 10,97 & 0,58 & 0,53 & 0,24 & 0,25 & 8,40 & 0,35 & 0,09 & 0,00 & 1,00 \\
\hline \multicolumn{11}{|l|}{ Triatoma brasiliensis } \\
\hline $\mathrm{n}$ & 85 & 4.865 & 33.880 & 34 & 4.238 & 2.683 & 8.218 & 1.477 & 0 & 55.480 \\
\hline$\% *$ & 15,04 & 10,07 & 55,68 & 4,09 & 62,01 & 32,53 & 61,77 & 43,12 & 0,00 & 38,92 \\
\hline \multicolumn{11}{|l|}{ Triatoma pseudomaculata } \\
\hline $\mathrm{n}$ & 388 & 10.361 & 25.185 & 45 & 1.941 & 3.825 & 4.632 & 1.352 & 183 & 47.912 \\
\hline$\%$ * & 68,67 & 21,45 & 41,39 & 5,41 & 28,40 & 46,38 & 34,81 & 39,47 & 100,0 & 33,61 \\
\hline \multicolumn{11}{|l|}{ Triatoma sordida } \\
\hline $\mathrm{n}$ & 0 & 32.063 & 0 & 0 & 0 & 1 & 225 & 0 & 0 & 32.289 \\
\hline$\% *$ & 0,00 & 66,38 & 0,00 & 0,00 & 0,00 & 0,01 & 1,69 & 0,00 & 0,00 & 22,65 \\
\hline \multicolumn{11}{|l|}{ Outras } \\
\hline $\mathrm{n}$ & 30 & 150 & 1.449 & 751 & 638 & 1.038 & 169 & 593 & 0 & 4.828 \\
\hline$\%$ & 5,32 & 0,31 & 2,38 & 90,26 & 9,34 & 12,59 & 1,27 & 15,74 & 0,00 & 3,39 \\
\hline Total & 565 & 48.305 & 60.847 & 832 & 6.834 & 8.247 & 13.305 & 3.425 & 183 & 142.543 \\
\hline$\%$ no Nordeste & 0,44 & 33,9 & 42,69 & 0,58 & 4,79 & 5,79 & 9,33 & 2,40 & 0,12 & \\
\hline $\begin{array}{l}\text { Densidade/unidade } \\
\text { doméstica pesquisada }\end{array}$ & 0,04 & 0,20 & 0,22 & 0,03 & 0,14 & 0,15 & 0,15 & 7,09 & 0,03 & \\
\hline
\end{tabular}

* sobre o total de capturas no estado

$\mathrm{n}=$ Número

Fonte: Gerência Técnica de Doença de Chagas, Centro Nacional de Epidemiologia, Fundação Nacional de Saúde. 
gipe e Rio Grande do Norte, com resíduos mínimos ocorrendo no Ceará, na Bahia, no Piauí, no Ceará, na Paraíba, no Maranhão e no Piauí. Grosseiramente, as capturas desta espécie caíram em todos os estados nordestinos entre 1979 e 1997, mantendo-se cerca de zero no Rio Grande do Norte.

Ao contrário, as capturas de $T$. brasiliensis e T. pseudomaculata aumentaram proporcionalmente do inquérito de 1979 para os dados de 1997, especialmente a última, assim como o se verifica para outras espécies (Tabelas 5 e 7). Para T. brasiliensis e T. pseudomaculata, na corrente década, observa-se uma certa alternância de predomínio em alguns Estados, em diferentes momentos, ainda que o número de insetos capturados tenda concretamente a decair nas áreas trabalhadas de maneira contínua pela FNS (Ceará, Paraíba, Rio Grande do Norte e Sergipe), ainda que uma certa tendência à ascensão tenha se registrado na Bahia e em Pernambuco. É particularmente importante uma avaliação da FNS/Ceará, que acompanhou minuciosamente a evolução dos índices de infestação e infecção natural de $T$. brasiliensis em Quixerê, Ceará, mantida a regularidade do controle químico na área: de 1979 a 1994, a infestação predial reduziu-se de $18 \%$ para $4 \%$; a densidade de insetos por vivenda examinada desceu de 1,37 para 0,16 e a taxa de infecção natural de $13,2 \%$ para $0,4 \%$ no mesmo período. Isso mostra que a espécie responde ao controle quando este mantém a necessária continuidade, mesmo em se tratando de uma espécie ubiqüista e com grande presença no ambiente silvestre peridomiciliar.

Nos dados disponíveis para 1998, já se divisa a enorme redução de $T$. infestans e $P$. megistus em paralelo com baixa densidade de triatomíneos por casa investigada no Nordeste, em volta de 0,18 inseto por casa pesquisada. Excluindo-se o Rio Grande do Norte (com baixíssimo número de unidades pesquisadas em 1998 , as maiores densidades ocorreram no Ceará $(0,22$ inseto/unidade investigada) e Bahia $(0,20)$, sendo as menores densidades registradas em Sergipe e Maranhão $(0,03)$ e em Alagoas $(0,04)$ (Tabela 7).

Para T. sordida, o quadro mostra o inseto basicamente na Bahia, também aparecendo em outros Estados, sem aparentar domiciliação importante em nenhum deles (Tabela 5).

Registra-se, ainda, que os índices de capturas intradomiciliares no Nordeste são discretos, segundo a FNS, exceção feita ao T. infestans na Bahia, ao T. rubrofasciata no Maranhão (praticamente restritas a São Luís) e a algumas áreas de T. brasiliensis no Ceará e no Piauí. Via
Tabela 7

Número de Triatoma brasiliensis, Triatoma pseudomaculata, e Panstrongylus megistus capturados por ano e por estado do Nordeste brasileiro entre 1993 e 1997, pela Fundação Nacional de Saúde.

\begin{tabular}{|c|c|c|c|c|c|}
\hline Estado/Espécie & 1993 & 1994 & 1995 & 1996 & 1997 \\
\hline \multicolumn{6}{|l|}{ Alagoas } \\
\hline T. brasiliensis & 2 & 17 & 28 & 91 & 85 \\
\hline T. pseudomaculata & 6 & 36 & 118 & 472 & 388 \\
\hline P. megistus & 313 & 283 & 102 & 7 & 62 \\
\hline \multicolumn{6}{|l|}{ Bahia } \\
\hline T. brasiliensis & 968 & 294 & 3.046 & 5.466 & 4.865 \\
\hline T. pseudomaculata & 5.570 & 3.998 & 7.280 & 12.281 & 10.361 \\
\hline P. megistus & 430 & 417 & 455 & 843 & 281 \\
\hline \multicolumn{6}{|l|}{ Ceará } \\
\hline T. brasiliensis & 50.196 & 18.561 & 22.723 & 32.508 & 33.880 \\
\hline T. pseudomaculata & 41.449 & 12.389 & 24.606 & 29.921 & 25.185 \\
\hline P. megistus & 2.188 & 1.036 & 1.014 & 466 & 323 \\
\hline \multicolumn{6}{|l|}{ Maranhão } \\
\hline T. brasiliensis & 31 & 29 & 30 & 17 & 34 \\
\hline T. pseudomaculata & 20 & 17 & 21 & 61 & 45 \\
\hline P. megistus & 6 & 1 & 8 & 1 & 2 \\
\hline \multicolumn{6}{|l|}{ Paraíba } \\
\hline T. brasiliensis & 6.039 & 3.037 & 11.565 & 4.346 & 4.238 \\
\hline T. pseudomaculata & 6.336 & 1.899 & 10.502 & 4.871 & 1.941 \\
\hline P. megistus & 163 & 12 & 115 & 15 & 17 \\
\hline \multicolumn{6}{|l|}{ Pernambuco } \\
\hline T. brasiliensis & 1.143 & 2.215 & 3.112 & 2.430 & 2.663 \\
\hline T. pseudomaculata & 1.751 & 1.604 & 3.801 & 4.803 & 3.825 \\
\hline P. megistus & 2.462 & 931 & 1.746 & 372 & 693 \\
\hline \multicolumn{6}{|l|}{ Piauí } \\
\hline T. brasiliensis & 7.824 & 11.256 & 14.734 & 25.984 & 8.218 \\
\hline T. pseudomaculata & 5.018 & 4.082 & 7.366 & 12.880 & 4.632 \\
\hline P. megistus & 21 & 3 & 40 & 16 & 47 \\
\hline \multicolumn{6}{|l|}{ Rio Grande do Norte } \\
\hline T. brasiliensis & 5.452 & 4.304 & 4.535 & 4.028 & 1.477 \\
\hline T. pseudomaculata & 7.974 & 3.967 & 5.147 & 6.760 & 1.352 \\
\hline P. megistus & 12 & 1 & 3 & $\mathrm{NI}$ & 3 \\
\hline \multicolumn{6}{|l|}{ Sergipe } \\
\hline T. brasiliensis & 0 & 2 & 0 & 0 & 0 \\
\hline T. pseudomaculata & 478 & 1.130 & 483 & 154 & 183 \\
\hline P. megistus & 10 & 121 & 76 & 5 & $\mathrm{NI}$ \\
\hline
\end{tabular}

$\mathrm{NI}=$ Não informado. 
de regra, entre cerca de $60 \%$ e $80 \%$ das capturas nos últimos anos têm sido peridomiciliares. Já para T. pseudomaculata e T. sordida, essa proporção é ainda maior, correspondendo a 95\% ou mais, segundo a Coordenação de Controle de Vetores/Centro Nacional de Epidemiologia/Fundação Nacional de Saúde/Brasília (CCDTV/CENEPI/FNS).

No conjunto, a dispersão dos triatomíneos capturados pela FNS ainda apresenta níveis médio-altos, chegando a quase $29 \%$ em 1997 , consideradas as informações de 249 municípios de quatro Estados. No todo, a infestação predial foi relativamente baixa $(4,44 \%)$ nas 579.956 unidades domiciliares pesquisadas. No Ceará, com o PCDCh mais atuante e concentrado em áreas de maior endemicidade, a dispersão alcançou $63,82 \%$ e a infestação domiciliar 8,84\% (Tabela 8).

No tocante às taxas de infecção natural pelo T. cruzi, há grande variação por espécie, época e local de captura, no geral sendo mais altas no passado, para $P$. megistus e T. infestans, com um nível intermédio para $T$. brasiliensis e taxas significativamente menores para T. pseudomaculata e T. sordida. Assim, olhando-se como um todo, a taxa de infecção natural de 266.906 triatomíneos examinados no Nordeste entre 1975 e 1982 foi de $3,37 \%$, contra $1,47 \%$ entre 78.841 examinados em 1997/98, conforme a CCDTV/ FNS. A Tabela 9 explicita esses dados para os Estados nordestinos com maior volume de capturas, mostrando ainda a evolução das taxas de infecção natural dos principais triatomíneos capturados na Bahia, Pernambuco e Ceará, os estados com PCDCh mais atuante nos últimos anos. Observa-se uma redução da positividade natural dos triatomíneos capturados pela FNS com relação aos dados de 1978-1980, tanto no total da região como nos três principais Estados analisados na Tabela 8.

Na Tabela 10, relativa ao ano de 1996, observa-se a variação das taxas de infecção natural entre os Estados do Nordeste e entre as grandes regiões do Brasil, como um todo, conforme os dados da FNS, ressaltando-se a maior proporção de capturas no Nordeste e seu índice global de infecção natural mais alto que o do País, apenas inferior ao da Região Sul. Nota-se que o discrepante índice de 35,81\% no Maranhão corresponde basicamente a T. rubrofasciata. Em particular, os dados para T. brasiliensis, T. pseudomaculata e T. infestans são mostrados na Tabela 11 para 1997, indicando maior positividade para T. infestans em comparação às outras duas, além da franca tendência à redução da positividade desses triatomíneos em relação ao inquérito realizado entre 1975 e 1983. Relativamente ao T. infestans, cujas capturas no Nordeste corresponderam a $64 \%$ das capturas do país em 1997, sua taxa de infecção natural naquele ano foi menor no Nordeste $(2,17 \%)$ que para o Brasil (2,90\%) É de se registrar ainda que essa taxa no País decresceu progressiva-

Tabela 8

Graus de infestação triatomínica por município e taxas de dispersão e infestação predial por triatomíneos em 1998, por estado do Nordeste brasileiro, em 1997.

\begin{tabular}{|c|c|c|c|c|c|c|c|c|c|c|}
\hline & $A L$ & BA & CE & MA & PB & PE & PI & RN & SE & Total \\
\hline \multicolumn{11}{|l|}{ Municípios } \\
\hline Pesquisados & $\mathrm{NI}$ & 111 & 96 & 14 & $\mathrm{NI}$ & 20 & $\mathrm{NI}$ & $\mathrm{NI}$ & 8 & 249 \\
\hline Positivos & - & 35 & & 4 & - & - & - & - & 7 & 46 \\
\hline$\%$ & - & 31,53 & 0 & 28,57 & - & - & - & - & 87,5 & 18,47 \\
\hline \multicolumn{11}{|l|}{ Localidades } \\
\hline Pesquisadas & - & 10.306 & 7.586 & 155 & - & - & - & - & 121 & 18.168 \\
\hline Positivas & - & 147 & 4.841 & 17 & - & - & - & - & 57 & 5.062 \\
\hline \% (dispersão) & - & 1,43 & 63,82 & 10,99 & - & - & - & - & 47,11 & 27,86 \\
\hline \multicolumn{11}{|c|}{ Unidades domiciliares } \\
\hline Pesquisadas & 14.088 & 246.286 & 282.050 & 30.583 & - & - & - & - & 6.949 & 579.956 \\
\hline Positivas & 364 & 291 & 24.943 & 61 & - & - & - & - & 108 & 25.767 \\
\hline \% (infestação) & 2,58 & 0,12 & 8,84 & 0,20 & - & - & - & - & 1,55 & 4,44 \\
\hline
\end{tabular}

$\mathrm{NI}=$ não informado.

Fonte: Gerência Técnica de Doença de Chagas, Centro Nacional de Epidemiologia, Fundação Nacional de Saúde. 
Índice de infecção natural dos triatomíneos capturados, por estado, no Nordeste brasileiro.

\begin{tabular}{|c|c|c|c|c|c|c|c|c|c|c|}
\hline & $A L$ & BA & CE & MA & PB & PE & PI & RN & SE & Total \\
\hline \multicolumn{11}{|l|}{$1978 / 1980$} \\
\hline Examinados & 16.970 & 58.102 & 53.636 & $\mathrm{NI}$ & 14.941 & 17.937 & 15.966 & 79.014 & 10.340 & 266.906 \\
\hline Infectados (\%) & 2,10 & 4,20 & 6,60 & $\mathrm{NI}$ & 3,30 & 4,40 & 4,40 & 0,80 & 0,40 & 3,37 \\
\hline \multicolumn{11}{|l|}{ 1997/1998 } \\
\hline Capturados & $\mathrm{NI}$ & 48.305 & 60.847 & 655 & $\mathrm{NI}$ & 12.660 & $\mathrm{NI}$ & $\mathrm{NI}$ & 258 & 122.725 \\
\hline Examinados & $\mathrm{NI}$ & 28.552 & 36.974 & 655 & $\mathrm{NI}$ & 12.660 & $\mathrm{NI}$ & $\mathrm{NI}$ & $\mathrm{NI}$ & 78.841 \\
\hline Infectados & $\mathrm{NI}$ & 357 & 267 & 233 & $\mathrm{NI}$ & 302 & $\mathrm{NI}$ & $\mathrm{NI}$ & $\mathrm{NI}$ & 1.159 \\
\hline Infectados (\%) & $\mathrm{NI}$ & 1,25 & 0,72 & 35,57 & $\mathrm{NI}$ & 2,39 & $\mathrm{NI}$ & $\mathrm{NI}$ & $\mathrm{NI}$ & 1,47 \\
\hline
\end{tabular}

$\mathrm{NI}=$ não informado

mente de $8,7 \%$ em 1979 para 3,41 em 1993, $2,16 \%$ em 1995 e 3,78 em 1996, finalmente alcançando os 2,90\% em 1997 (dados da Gerência Técnica de Chagas, FNS, Brasília).

Com relação ao grau de cobertura do PCDCh na região nordestina, de modo geral, tem havido, como no resto do País, uma evidente redução da força do programa, mercê de uma progressiva diminuição da força de trabalho na FNS, em particular ao longo da década de 90 . A Tabela 12 explicita o número de unidades domiciliares pesquisadas pela FNS em cada estado e na região, entre 1993 e 1997, observandose a redução global do trabalho e sua relativa estabilidade em alguns estados, como a Bahia (onde a prioridade tem sido mantida, a duras penas, no combate ao T. infestans). No entanto, de modo geral, a atividade tem decrescido, sendo mais sacrificadas aquelas áreas passadas à vigilância epidemiológica, segundo os informes dos estados à Gerência de Doença de Chagas da FNS em Brasília.

Um outro aspecto importante no Nordeste refere-se à detecção de focos periurbanos de triatomíneos, em várias cidades do Ceará (Crateús, Sobral, Independência), do Piauí (Picos, São Raimundo Nonato), de Pernambuco (Sertânia, Floresta), na Bahia (Bom Jesus da Lapa, Barreiras), etc., geralmente nas periferias urbanas, priorizando os peridomicílios e apresentando baixas densidades de infestação.

\section{Infecção humana}

No inquérito nacional de 1978-1980, a então SUCAM detectou $3,05 \%$ de prevalência da infecção chagásica na população geral rural do Nordeste, sendo as maiores cifras para a Bahia $(7,40 \%)$ e as menores para o Maranhão $(0,20 \%)$.

\begin{tabular}{|c|c|c|}
\hline \multicolumn{3}{|c|}{$\begin{array}{l}\text { Infecção natural pelo Trypanosoma cruzi de } \\
\text { triatomíneos capturados nos estados do Nordeste, } \\
\text { no Brasil e nas grandes regiões brasileiras, em } 1996 .\end{array}$} \\
\hline & $\begin{array}{l}\text { Número de } \\
\text { capturados }\end{array}$ & $\begin{array}{l}\text { \% de } \\
\text { infecção }\end{array}$ \\
\hline Maranhão & 1.240 & 35,81 \\
\hline Piauí & 43.100 & 0,63 \\
\hline Ceará & 64.714 & 0,40 \\
\hline Rio Grande do Norte & 13.735 & 2,61 \\
\hline Paraíba & 10.340 & 2,42 \\
\hline Pernambuco & 8.431 & 2,43 \\
\hline Alagoas & 594 & 1,00 \\
\hline Sergipe & 160 & 0,00 \\
\hline Bahia & 58.842 & 1,22 \\
\hline Nordeste & 201.156 & 1,23 \\
\hline Norte & 2.665 & 0,70 \\
\hline Sul & 2.291 & 2,00 \\
\hline Sudeste & 69.832 & 0,53 \\
\hline Centro-Oeste & 14.632 & 0,63 \\
\hline Brasil & 290.576 & 1,03 \\
\hline
\end{tabular}

Com o avanço do PCDCh, um inquérito sorológico entre 122.027 escolares do Nordeste mostrou uma prevalência de apenas $0,08 \%$ da esquizotripanose, sem dúvida, espelhando significativa redução em relação aos dados de vinte anos passados (Tabela 13). Em particular, uma visão atual da curva de prevalência por grupos de idade foi dada por Diotaiuti et al. (1998), ao analisar 2.450 pessoas da população geral de Independência, Ceará, área de infestação ainda importante por T. brasiliensis e T. pseudomaculata. Da prevalência geral de 5,7\% de in- 
Infecção natural por Trypanosoma cruzi de Triatoma brasiliensis, Triatoma pseudomaculata e Triatoma infestans capturados no Nordeste do Brasil em 1997 e no inquérito triatomínico (Brasil) 1978-1980.

\begin{tabular}{lccrc}
\hline Período & T. brasiliensis & T. pseudomaculata & T. infestans & Totais \\
\hline 1997/Nordeste & & & & \\
no capturados & 55.513 & 48.145 & $686^{* *}$ & 104.344 \\
no examinados & 35.662 & 34.467 & 415 & 70.544 \\
no positivos & 514 & 444 & 9 & 967 \\
\% positivos & 1,44 & 1,29 & 1,37 \\
Inquérito 1978-1980/Brasil & & & & 387.615 \\
no capturados & 99.845 & 125.634 & 1,76 & - \\
\% positivos & 6,7 & 1,7 & 8,7 \\
\hline
\end{tabular}

* Fonte: Gerência Técnica de Doença de Chagas, Centro Nacional de Epidemiologia,

Fundação Nacional de Saúde e Castro Filho \& Silveira, 1984.

** Destes, $85 \%$ na Bahia e $11 \%$ na Paraíba, estando os $4 \%$ restantes em Pernambuco e Piauí.

Tabela 12

Número de unidades domiciliares pesquisadas pela Fundação Nacional de Saúde, por estado e por ano, entre 1993 e 1997.

\begin{tabular}{lrrrrrrrrrrr}
\hline $\begin{array}{l}\text { Número de unidades } \\
\text { domiciliares pesquisadas } \\
\text { por ano }\end{array}$ & AL & BA & CE & MA & PB & PE & PI & RN & SE & Nordeste \\
\hline 1993 & 37.977 & 236.706 & 635.583 & 37.828 & 160.696 & 154.764 & 67.753 & 65.420 & 43.226 & 1.441 .946 \\
1994 & 34.149 & 171.125 & 243.466 & 36.072 & 67.672 & 93.531 & 92.428 & 84.566 & 69.719 & 894.722 \\
1995 & 18.939 & 82.773 & 358.418 & 57.972 & 154.382 & 106.068 & 121.793 & 89.918 & 28.097 & 1.020 .355 \\
1996 & 12.973 & 190.930 & 341.201 & 47.469 & 75.841 & 62.805 & 130.154 & 56.299 & 8.887 & 928.555 \\
1997 & 14.088 & 247.287 & 364.904 & 34.662 & 50.476 & 55.119 & 89.169 & 483 & 4.284 & 862.469 \\
\hline
\end{tabular}

Fonte: Silveira \& Vinhaes, 1998.

fecção chagásica, apenas $0,8 \%$ ocorre nos indivíduos menores de dez anos, crescendo a prevalência até a faixa dos cinqüenta anos ou mais, com 12,6\% (Tabela 14). De forma similar, uma tendência à evolução regressiva nos índices de doadores chagásicos nos bancos de sangue nordestinos reforçam o dados acima, conforme a Tabela 15, de espelhando uma tendência geral do País (Dias \& Schofield, 1998).

\section{Morbidade e mortalidade}

Como sabido, na região nordestina, ocorrem todas as principais formas clínicas da DCH, especialmente a cardiopatia e a esofagopatia crônicas, aparentemente com variações regionais de impacto médico-social. De maneira sumária e com as devidas restrições, o histórico do Departamento de Informática do SUS - DATA-
SUS/MS (Tabela 16) mostra a evolução numérica das internações anuais por DCH nos estados nordestinos e no Brasil, apresentando-se uma tendência regressiva e maior impacto em estados como a Bahia e Alagoas. No entanto, os mesmos dados mostram que, no Nordeste, registraram-se apenas $4,40 \%$ das internações por DCH do país, apresentando uma taxa de internação de apenas 0,063/100.000 habitantes, em comparação à de 5,763/100.000 habitantes do Brasil, no ano de 1997. Já em termos do registro de mortalidade, igualmente, dados de 1997 refletem um menor impacto aparente no Nordeste (1,82 óbitos/DCH/100.000 habitantes) em relação a 3,38/100.000 para o Brasil. A Tabela 17 explicita esses dados por estados nordestinos apontando, grosso modo, maior impacto aparente também para a Bahia (índice de 4,26 óbitos por DCH/100.000 habitantes) e 
Nordeste do Brasil: inquérito sorológico em escolares em 1997/1998 e da população geral rural em 1978/1980.

\begin{tabular}{|c|c|c|c|c|c|c|c|c|c|c|}
\hline & $A L$ & BA & CE & MA & PB & PE & PI & RN & SE & Total \\
\hline \multicolumn{11}{|l|}{$1997 / 1998$} \\
\hline Amostras processadas & 7.335 & 26.657 & 38.429 & 1.025 & 21.769 & 1.864 & 4.939 & 12.583 & 7.426 & 122.027 \\
\hline Positivos & 0 & 7 & 9 & 0 & 35 & 1 & 2 & 25 & 14 & 93 \\
\hline$\%$ & 0,00 & 0,03 & 0,02 & 0,00 & 0,16 & 0,05 & 0,04 & 0,20 & 0,19 & 0,08 \\
\hline \multicolumn{11}{|l|}{$1978 / 1980$} \\
\hline Amostras processadas & 10.193 & 58.969 & 29.973 & 51.529 & 42.484 & 53.193 & 35.420 & 29.941 & 20.777 & 332.779 \\
\hline Positivos & 520 & 4.364 & 390 & 103 & 977 & 1.117 & 1.322 & 359 & 9.970 & 10.149 \\
\hline$\%$ & 5,10 & 7,40 & 1,30 & 0,20 & 2,30 & 2,10 & 3,70 & 1,20 & 4,80 & 3,05 \\
\hline
\end{tabular}

Fonte: Gerência Técnica de Doença de Chagas, Centro Nacional de Epidemiologia, Fundação Nacional de Saúde.

os menores para Sergipe e Maranhão, próximos a zero.

É importante notar que, apesar de ser, atualmente, a região com maior número de capturas de triatomíneos no Brasil, o Nordeste registra apenas $0,37 \%$ de seus óbitos por DCH, o que equivale a somente $14,27 \%$ das mortes registradas pela doença no País. Para cotejar os dados de mortalidade e da assistência médica, em geral, a mesma tabela mostra que o Nordeste registra $23,11 \%$ das mortes por doenças infecciosas e parasitárias do país, apenas $14,05 \%$ das mortes por neoplasias e, praticamente, a metade dos óbitos ocorre sem assistência médica.

\section{Discussão}

No Nordeste, o grande número de espécies chama a atenção, indicando diversidade ambiental e ação antrópica. Nessa parte do País, estão assinaladas diferentes regiões fisiográficas que comportam espécies bem diferentes entre si, assim como floresta tropical e subtropical (albergando T. sordida, T. vitticeps, P. diasi, P. lutzi, P. megistus), savanas (cerrados e caatingas, albergando $P$. tertius, T. infestans, T. rubrofasciata, T. sordida), florestas secas, tropicais e subtropicais (albergando P. tertius, T. costalimai, $T$. pseudomaculata, T. sordida, P. megistus e P. geniculatus) e florestas xerofíticas (com T. brasiliensis, T. costalimai, T. infestans, T. sordida, P. diasi e P. geniculatus) (Forattini, 1980; Schofield, 1994; Carcavallo et al., 1997).

As 27 (ou 29) espécies assinaladas no Nordeste correspondem a mais de $50 \%$ das registradas no Brasil, seja nas capturas intradomiciliares da SUCAM/FNS, seja nos trabalhos de pesquisa acadêmica (Lent \& Wigodzinsky, 1979;

\begin{tabular}{|c|c|c|}
\hline \multicolumn{3}{|c|}{$\begin{array}{l}\text { Prevalência da infecção chagásica em população } \\
\text { geral não selecionada de Independência, Ceará, } \\
\text { em } 1998 .\end{array}$} \\
\hline $\begin{array}{l}\text { Grupos de } \\
\text { idade (anos) }\end{array}$ & $\begin{array}{l}\text { Número de } \\
\text { examinados }\end{array}$ & $\%$ positivos \\
\hline $0-10$ & 657 & 0,8 \\
\hline $11-20$ & 547 & 3,5 \\
\hline $21-30$ & 337 & 5,6 \\
\hline $31-40$ & 242 & 7,0 \\
\hline $41-50$ & 207 & 10,6 \\
\hline 50 e mais & 460 & 12,6 \\
\hline Total & 2.450 & 5,7 \\
\hline
\end{tabular}

Fonte: Diotaiuti et al., 1998.

Carcavallo et al., 1997; Silveira \& Vinhaes, 1998). Há que se considerar, nesses dados, que a imensa maioria dos insetos procede da SUCAM/FNS, correspondendo, portanto, a capturas e/ou notificações de âmbito domiciliar (intra ou peridomicílio).

Sobre a detecção eventual de R. prolixus na região, a identificação exata da espécie nunca ficou bem definida, cabendo ao próprio Neiva (1944) advertir que o assunto deveria ficar em suspenso, pois a sistemática do gênero era complicada e as possibilidades de confusão com outras espécies (notadamente $R$. nasutus) era concreta. Não obstante, $R$. prolixus foi detectado posteriormente em peridomicílios e palmeiras de Tocantins (Diotaiuti et al., 1984), assim como assinalado por Carcavallo \& Tonn (1984) nos Estados do Amazonas e do Pará, sendo perfeitamente possível, portanto, que a espécie já 
Percentual de sorologia positiva na hemorrede pública do Nordeste por Unidade Federativa. Brasil 1987/1994.

\begin{tabular}{lrrrrrrrr}
\hline Unidade Federativa & \multicolumn{7}{c}{ Prevalência entre doadores } \\
& 1987 & 1988 & 1989 & 1990 & 1991 & 1992 & 1993 & $1994^{*}$ \\
\hline Alagoas & 3,00 & 3,69 & 0,14 & 2,23 & 0,44 & 5,79 & 4,80 & 0,80 \\
Bahia & 1,60 & 1,30 & 2,22 & 1,69 & 0,77 & 1,48 & 1,05 & 0,90 \\
Ceará & 0,39 & 0,87 & 0,34 & 0,18 & 0,12 & 0,67 & 0,38 & 0,35 \\
Maranhão & 0,02 & 0,05 & 0,12 & 0,06 & 0,08 & 0,02 & 0,00 & 0,14 \\
Paraíba & $\mathrm{NI}$ & $\mathrm{NI}$ & $\mathrm{NI}$ & $\mathrm{NI}$ & 0,00 & 0,07 & 0,15 & 0,00 \\
Pernambuco & 0,22 & 0,30 & 0,20 & 0,20 & 0,10 & 0,10 & 0,11 & 0,14 \\
Piauín & 0,18 & 0,07 & 0,09 & 0,17 & 0,20 & 0,07 & 0,07 & 0,24 \\
Rio Grande do Norte & $\mathrm{NI}$ & 0,47 & 1,37 & 1,08 & 0,90 & 0,30 & 0,24 & 0,39 \\
Sergipe & 0,47 & 0,25 & 0,31 & 0,46 & 0,56 & 0,18 & 0,52 & 0,17 \\
Nordeste & 0,84 & 0,88 & 0,60 & 0,76 & 0,35 & 0,96 & 0,46 & 0,36 \\
Brasil & 0,98 & 0,88 & 0,79 & 0,73 & 0,76 & 0,80 & 0,69 & 0,75 \\
\hline
\end{tabular}

Fonte: Coordenação Nacional de Sangue e Hemoderivados, Secretaria de Ações da Saúde, Ministério da Saúde. * 1o semestre/1994

$\mathrm{NI}=\mathrm{Não}$ informado.

Tabela 16

Internações por doença de Chagas no Nordeste do Brasil, por estado. 1990-1997.

\begin{tabular}{|c|c|c|c|c|c|c|c|c|c|}
\hline Estado & 1990 & 1991 & 1992 & 1993 & 1994 & 1995 & 1996 & 1997 & $\begin{array}{l}\text { Total } \\
1990-97\end{array}$ \\
\hline Alagoas & 13 & 14 & 47 & 35 & 24 & 6 & 10 & 4 & 153 \\
\hline Bahia & 15 & 26 & 37 & 30 & 38 & 23 & 18 & 13 & 200 \\
\hline Ceará & 4 & 5 & 2 & 1 & 0 & 0 & 2 & 4 & 18 \\
\hline Maranhão & 2 & 1 & 1 & 1 & 8 & 5 & 1 & 0 & 19 \\
\hline Paraíba 1989/95 & 6 & 9 & 6 & 3 & 3 & 4 & 1 & 0 & 32 \\
\hline Pernambuco & 5 & 5 & 1 & 1 & 2 & 2 & 7 & 3 & 26 \\
\hline Piauí & 5 & 3 & 2 & 0 & 2 & 0 & 0 & 0 & 12 \\
\hline Rio Grande do Norte & 0 & 4 & 0 & 1 & 1 & 1 & 2 & 2 & 11 \\
\hline Sergipe & 2 & 0 & 0 & 0 & 2 & 0 & 1 & 1 & 6 \\
\hline Total & 52 & 67 & 96 & 72 & 80 & 41 & 42 & 27 & 477 \\
\hline Brasil & 1.836 & 1.838 & 1.657 & 1.441 & 1.232 & 966 & 951 & 922 & 10.843 \\
\hline \% sobre o Brasil & 2,83 & 3,65 & 5,79 & 5,00 & 6,49 & 4,24 & 4,42 & 2,93 & 4,40 \\
\hline
\end{tabular}

Fonte: Gerência Técnica de Doença de Chagas, Centro Nacional de Epidemiologia, Fundação Nacional de Saúde.

tenha aparecido ou venha a aparecer no Nordeste, em princípio em focos de palmeiras.

Já para o T. rubrovaria na Bahia, o registro é único e esporádico, com certeza, em virtude do transporte passivo de algum exemplar proveniente de migrações gaúchas, como, de regra, se registra para Rondônia, não significando sequer a introdução da espécie, muito menos sua colonização. As diferenças fundamentais de comportamento e distribuição das espécies de triatomíneos no Nordeste brasileiro se explicam pe- la ecologia de cada microregião, pelo tipo e intensidade da ação antrópica e pela capacidade de domiciliação e colonização de cada espécie, o que resulta, finalmente, na definição de sua importância epidemiológica em relação à DCH.

Assim, embora com um número elevado de espécies registrado ao longo da história, aquelas mais importantes do Nordeste são, na prática, muito poucas, restringindo-se ao T. brasiliensis, P. megistus e T. infestans (espécies básicas), sendo imediatamente secundárias T. pseu- 
Mortalidade geral para o Brasil, Nordeste e estados do Nordeste, em 1997, segundo doenças infecciosas e parasitárias, doença de Chagas, neoplasias (todas) e óbitos sem assistência médica.

\begin{tabular}{|c|c|c|c|c|c|c|c|c|c|}
\hline \multirow[t]{2}{*}{$\begin{array}{l}\text { Grandes regiões e } \\
\text { Unidades Federativas }\end{array}$} & \multirow[t]{2}{*}{ Total de óbitos } & \multicolumn{2}{|c|}{$\begin{array}{l}\text { Doenças infecciosas } \\
\text { e parasitárias }\end{array}$} & \multicolumn{2}{|c|}{ Doença de Chagas } & \multicolumn{2}{|c|}{ Neoplasias (Total) } & \multicolumn{2}{|c|}{$\begin{array}{l}\text { Óbitos sem } \\
\text { assistência médica }\end{array}$} \\
\hline & & $n$ & $\%$ & $n$ & $\%$ & $\mathrm{n}$ & $\%$ & $n$ & $\%$ \\
\hline Brasil & 903.516 & 47.936 & 5,31 & 5.410 & 0,60 & 106.990 & 11,84 & 86.724 & 9,60 \\
\hline Nordeste & 206.612 & 11.079 & 5,36 & 772 & 0,37 & 15.028 & 7,27 & 41.608 & 20,14 \\
\hline \% Nordeste/Brasil & 22,87 & 23,11 & & 14,27 & & 14,05 & & 47,98 & \\
\hline Maranhão & 13.462 & 524 & 3,89 & 0 & 0,00 & 744 & 5,53 & 4.414 & 32,79 \\
\hline Piauí & 7.743 & 338 & 4,37 & 34 & 0,44 & 565 & 7,30 & 2.297 & 29,67 \\
\hline Ceará & 31.160 & 2.159 & 6,93 & 35 & 0,11 & 2.910 & 9,34 & 6.218 & 19,96 \\
\hline Rio Grande do Norte & 12.040 & 563 & 4,68 & 8 & 0,07 & 1.060 & 8,80 & 3.014 & 25,03 \\
\hline Paraíba & 17.394 & 588 & 3,38 & 10 & 0,06 & 802 & 4,61 & 8.326 & 47,87 \\
\hline Pernambuco & 49.532 & 2.712 & 5,48 & 120 & 0,24 & 3.815 & 7,70 & 8.734 & 17,63 \\
\hline Alagoas & 14.279 & 793 & 5,55 & 67 & 0,47 & 691 & 4,84 & 5.095 & 35,68 \\
\hline Sergipe & 8.444 & 429 & 5,08 & 2 & 0,02 & 503 & 5,96 & 2.205 & 26,11 \\
\hline Bahia & 52.558 & 2.973 & 5,66 & 506 & 0,96 & 3.938 & 7,49 & 13.075 & 24,88 \\
\hline
\end{tabular}

Fonte: DATASUS, 1998.

domaculata e R. nasutus. T. sordida é uma espécie muito capturada na Bahia, mas sempre em situações peridomiciliares e com taxas de infecção natural que nunca excedem $0,8 \%$, sem tendência aparente a subir, nos últimos vinte anos (Silveira \& Vinhaes, 1998). Aliás, mesmo se mantendo há anos como a espécie mais capturada pelo PCDCh no Brasil, T. sordida segue tendo características de pouca periculosidade quanto à transmissão da DCH, tanto por seu comportamento basicamente peridomiciliar como por sua marcada ornitofilia e baixos índices de infecção natural pelo T. cruzi. Tem-se visto que, nas regiões onde predomina essa espécie, os índices de prevalência da infecção chagásica entre humanos é mínima ou ausente, fato verificável na Bahia, Goiás, Minas Gerais e São Paulo (Diotaiuti et al., 1993; Rocha-e-Silva et al., 1998; Silveira \& Vinhaes, 1998).

Das três espécies básicas, a T. brasiliensis é muito mais dispersa e abundante, justamente por suas características de espécie euritrópica, pouco higrófila e capaz de suportar temperaturas muito elevadas, portanto, aclimatada à grande extensão das terras nordestinas (Alencar, 1987). P. megistus, ao contrário, espécie estenotópica, higrófila e estenotérmica, restringe-se a microregiões e microambientes umbrosos e mais úmidos, mais raros e esparsos no Nordeste (mais ligados às zonas de mata, de onde se faz sua dispersão para ecótopos artificiais, havendo indícios de ter sido introduzida na região há muitas décadas (Forattini, 1980;
Pirajá-da-Silva, 1913). A evolução de P. megistus no Nordeste é aquela esperada para espécies introduzidas, basicamente domésticas e/ou restritas a ambientes em progressiva deterioração (ecótopos umbrosos e úmidos, em progressiva devastação no NE), refletindo ainda, como T. infestans, a intensidade e a cobertura do programa de controle (Forattini, 1980; Alencar, 1987; Schofield \& Dias, 1998). Assim, $P$. megistus está virtualmente desaparecendo do Nordeste brasileiro, haja vista a sua drástica redução absoluta e relativa em Estados onde já foi importante transmissora da DCH, como Bahia, Alagoas, Pernambuco e Ceará, o que se aquilata pelos dados apresentados e pelos registros da literatura (Castro Filho \& Silveira, 1984; Alencar, 1987; Silveira \& Vinhaes, 1998).

Um bom exemplo dessa eliminação foi documentado por Macedo (1976), que trabalhou em São Felipe, Bahia, onde $P$. megistus era abundante e infestava cerca de $23 \%$ das 2.522 casas pesquisadas, basicamente no intradomicilio, correspondendo a uma prevalência de $45 \%$ de doença de Chagas na população geral. Deflagrado o controle químico, pela então SUCAM, em 1985, já se reduzia a infestação a 9,3\%, com uma prevalência de $2,8 \%$ de DCH entre escolares. Atualmente, conforme a FNS/Bahia, a infestação em São Felipe é ausente, e a sorologia escolar apresenta-se totalmente negativa, atestando-se o sucesso do controle realizado.

Por seu turno, T. infestans foi uma espécie introduzida, que chegou passivamente ao Nor- 
deste por volta dos anos 50 e se dispersou com grande rapidez e intensidade entre casarios pobres a partir do sul, via Bahia, onde os maiores focos foram e continuam sendo detectados nos últimos vinte anos (Castro Filho \& Silveira, 1984; Silveira et al., 1984; Schofield \& Dias, 1998). Dado o seu peso epidemiológico, a relevância de T. infestans foi muito grande em vários Estados, mas seu combate - intensificado desde os anos 80 - resultou em sua virtual eliminação em vastas áreas, como se esperaria de uma espécie introduzida e exclusivamente domiciliar. A espécie está virtualmente eliminada do Nordeste, exceção aos focos do oeste da Bahia. Mesmo ali, os dados disponíveis na FNS indicam que as capturas estão se confinando e restringindo a duas dezenas de municípios, refletindo focos residuais que poderão se extinguir a curto ou médio prazo, se as ações de controle tiverem a necessária cobertura e continuidade (Schofield \& Dias, 1998; Silveira \& Vinhaes, 1998). Ao revés, e considerando-se o contexto presente, as possibilidades de um recrudescimento do T. infestans na região existem e fazem pressupor acurada vigilância epidemiológica, tendo em vista não somente o seu poder de dispersão a partir de focos residuais como também, especialmente, a manutenção da pobreza regional e o previsível desmonte da Fundação Nacional de Saúde no País, sem a devida absorção de suas atividades por Estados e municípios (Dias, 1991; Dias \& Coura, 1997; Schofield \& Dias, 1998).

No tocante aos índices de infecção natural dos triatomíneos capturados na região pelo $T$. cruzi, observa-se uma importante diversidade por espécie de triatomíneo e uma tendência à sua redução paulatina ao longo dos últimos vinte anos e em paralelo com a progressão do PCDCh, principalmente de T. infestans.. Esses fatos correspondem, em geral, ao potencial vetorial e comportamento das espécies, de um lado, e à dinâmica de transmissão do parasito, de outro, lembrando-se da clássica assertiva de Emmanuel Dias, de que representa este índice um verdadeiro "xenodiagnóstico natural" para uma determinada área num momento específico (Dias, 1936). Assim, pode-se estimar que a circulação do parasito no âmbito domiciliar do Nordeste, onde são capturados os triatomíneos pela FNS, tem paulatinamente se reduzido, conseqüentemente se reduzindo, também, os riscos de transmissão vetorial do T. cruzi ao homem (Alencar, 1987; Dias \& Coura, 1997; Schofield \& Dias, 1998).

O potencial vetorial dos triatomíneos em termos da transmissão da DCH depende de vários fatores, sendo principais o grau de domici- liação e colonização, o grau de antropofilia e os índices de infecção natural pelo T. cruzi (Neiva \& Lent, 1941; Forattini, 1980; Alencar, 1987; Schofield, 1994; Silveira \& Rezende, 1994; Dias \& Coura, 1997). Como bom exemplo, Alencar (1987) anota um gradiente desses fatores comparando P. megistus, T. brasiliensis e T. pseudomaculata de microregiões homogêneas do Ceará, encontrando maior prevalência da DCH para a área de P. megistus (mais domiciliado, maior antropofilia e $10,3 \%$ de infecção natural), prevalência um pouco menor para áreas dominadas por T. brasiliensis (menor colonização intradomiciliar e menor antropofilia, infecção natural de $13,7 \%$ ) e prevalência mínima na área de T. pseudomaculata (antropofilia e colonização intradomiciliar mínimas, infecção natural de 3,0\%). Dados semelhantes, para outras espécies, se encontram em outras regiões, como na América Central, onde a prevalência da DCH é significativamente maior nas áreas de $R$. prolixus (exclusivamente intradomiciliar, mais antropofílico e mais parasitado) que nas de T. dimidiata (intra ou peridomiciliar, menos antropofílico, menor infecção natural) (Schofield, 1994). A alternância histórica de espécies dominantes (Tabela 5) por Estado mostra um panorama dinâmico, com predominância de $T$. brasiliensis e T. pseudomaculata, em função das microregiões trabalhadas, assim como da ação antrópica ali ocorrida e da intensidade e continuidade do trabalho profilático realizado. Isso já fora assinalado por Alencar (1987), por Dias (1991) e por Forattini (1980), o que ratifica ainda mais a necessidade de vigilância (Dias, 1991).

Em particular, no caso de regiões onde existia em grande intensidade e foi combatido o $T$. infestans (no Nordeste, basicamente na Bahia) sua virtual eliminação vem dando margem ao aparecimento de outras espécies como T. sordida, como vem acontecendo no Sudeste (aumento relativo de $P$. megistus e T. sordida) ou no Sul do Brasil, com sua substituição por T. rubrovaria (Dias, 1991; Rocha-e-Silva et al., 1998, Schofield, 1994).

Já sobre a capacidade operacional do PCDCh na região, lamentavelmente, não cabe dúvida quanto à sua progressiva deterioração, o que compromete os resultados já alcançados e, principalmente, a sustentabilidade da vigilância epidemiológica (Dias, 1991, 1998; Schmunis \& Dias, neste mesmo fascículo). Por sinal, é preciso assinalar que a perda de fôlego da FNS quanto à luta antichagásica ocorre, ainda, por causa de outro fator de complicação desde o final da década passada, qual seja o crescimento da dengue e da cólera no Nordeste, resultando em desvios de pessoal e outros recursos para 
essas campanhas emergenciais (Dias, 1998; Silveira \& Vinhaes, 1998).

Os estudos de afinidade genética e comportamental entre as espécies auxiliam a compreender a distribuição das diferentes espécies na região, também podendo indicar, grosso modo, as perspectivas gerais de cada espécie numa área (Schofield, 1994; Dujardin et al., 1999). As pressões ecológicas e, particularmente, a ação antrópica explicam as alternâncias de espécies na região nordestina, ou mesmo a diversidade intra-específica que se observa há tempos com o próprio T. brasiliensis (Costa, 1999). Assim, no Maranhão, Estado com grande número de espécies detectadas, a DCH é a de menor importância na região, havendo poucas probabilidades de que venha agravar-se a endemia chagásica a curto ou médio prazo. Já na Bahia, caso não se eliminem definitivamente os focos residuais de T. infestans e P. megistus domiciliado, em paralelo com o funcionamento regular de vigilância epidemiológica, o risco de recrudescimento da DCH é bastante real.

Por outro lado, se a tendência historicamente observada de que T. sordida não representa maior perigo quanto à transmissão da doença de Chagas ao homem, esse risco para T. pseudomaculata e, especialmente, T. brasiliensis no Nordeste é mais palpável. Os dados indicam uma continuada e lenta expansão de T. pseudomaculata na região, desde os anos 70, mas sempre com menor domiciliação que $T$. brasiliensis, que tem mostrado padrões variáveis de infestação, até mesmo de redução da infestação domiciliar em alguns Estados, o que se vê na Tabela 7 e na literatura disponível (Castro Filho \& Silveira, 1984; Alencar, 1987; Silveira \& Vinhaes, 1998). Essas duas espécies (com exceção da área baiana onde remanesce T. infestans) representam o principal risco de transmissão vetorial da DCH no Nordeste atualmente e apresentam problemas práticos em seu controle. No conjunto, significam mais de $72 \%$ das capturas na região, seguidas por T. sordida, cujas capturas praticamente se restringem à Bahia (Tabela 6). De forma geral, seus focos são primordialmente peridomiciliares, com maior potencial invasivo para T. brasiliensis.

É oportuno referir que, no Ceará, estudos de Alencar (1987), entre 1978 e 1985, mostraram muito bem que o grau de antropofilia de T. pseudomaculata era de apenas $0,2 \%$ (espécie nitidamente ornitofílica, $78,4 \%$, praticamente restrita a cercas e galinheiros), sendo de $4,6 \%$ para $T$. brasiliensis (ornitofilia de 69,8\%, predominando no peridomicílio, mas encontrável no intradomicílio) e de $44,4 \%$ para $P$. megistus (ornitofilia de $53,9 \%$, capturada em intra e peridomicílio).
Já em relação à T. rubrofasciata, espécie doméstica por excelência, o principal foco remanesce na Ilha de São Luís, Maranhão, em densidades variáveis e com alta infecção natural, não parecendo boa transmissora da $\mathrm{DCH}$, haja vista a ausência de infecção humana nas áreas de captura. Há dificuldades em seu controle por tratar-se de área urbana muito dispersa e com ecótopos de alta complexidade. No entanto, a espécie pode ser eliminada, se houver necessidade e desejo político para tal (Schofield, 1994). O estudo do peridomicílio e da dinâmica triatomínica no seu âmbito tem sido um desafio para os pesquisadores, havendo neste mesmo suplemento um artigo que aborda o assunto (Oliveira-Lima et al.). Na maioria das vezes, os ecótopos peridomiciliares mais adequados à invasão e colonização dos triatomíneos são aqueles onde abrigo e alimento estão disponíveis, sempre predominando os galinheiros na maioria dos trabalhos disponíveis (Forattini, 1980; Alencar, 1987; Dias, 1991; Diotaiuti et al., 1993).

O manejo desses focos é difícil pela discreta atividade dos inseticidas nos mesmos e pela falta de prioridade à limpeza e higiene que lhes deveria dedicar a pobre população campesina (Dias, 1991, 1998). Por outro lado, há regiões onde a aridez é tanta e a disponibilidade de água e animais é tão escassa que os peridomicílios ficam pobres e a densidade triatomínica é menor, como foi verificado para focos peridomiciliares de T. sordida em Minas Gerais (Diotaiuti et al., 1993).

No caso do Nordeste, se no Ceará o trabalho contínuo da FNS tem ajudado a evitar a expansão de T. brasiliensis, no Piauí, onde a FNS praticamente só tem fôlego para operar os poucos municípios onde ocorreu T. infestans, as baixas prevalências da infecção humana em áreas de T. brasiliensis e T. pseudomaculata podem explicar-se pelo comportamento peridomiciliar e as densidades relativamente menores dessas espécies nas áreas socialmente mais deprimidas.

Já sobre o potencial vetorial e de colonização das demais espécies detectadas no nordeste, o acompanhamento dos dados e da literatura indicam baixa periculosidade, exceção, talvez, de $R$. nasutus, que tem aparecido com certa freqüência nos domicílios (Silveira \& Vinhaes, 1998).

Quanto aos focos periurbanos, estes têm sido detectados há décadas e resultam tanto da contínua migração rural-urbana na região como da pobreza e do aspecto semi-rural dos bairros periféricos de muitas cidades nordestinas, a exemplo do que ocorre em outros países, passando a constituir-se, progressivamente, em 
preocupação médico-sanitária (Alencar, 1987; Dias, 1991; Schofield, 1994; Dias \& Coura, 1997).

Finalmente, quanto ao fôlego do PCDCh na região, os dados e informações indicam que o progressivo esvaziamento da FNS, principalmente quanto à disponibilidade de recursos humanos, tem se feito sentir numa diminuição flagrante de cobertura, em particular ao longo da última década. Esse assunto é abordado em outro artigo do presente fascículo (Schmunis \& Dias) e na literatura (Dias, 1991, 1998; Schofield \& Dias, 1998; Silveira \& Vinhaes, 1998), refletindo uma grande preocupação dos técnicos e analistas quanto à necessária continuidade dos trabalhos que a região requer, para que não recrudesça a endemia chagásica na região. Trata-se de uma questão basicamente política e administrativa que envolve a descentralização e a tendência globalizante moderna, que privilegia o modelo econômico no "enxugamento" do Estado, conferindo responsabilidades e encargos às administrações locais e periféricas que - na maioria das vezes - não estão capacitadas, motivadas ou aparelhadas para fazê-lo (Dias, 1998).

Sobre a transmissão e a prevalência da DCH no Nordeste, os dados indicam uma situação de transição, com tendência à redução na região como um todo (Tabela 13). Observa-se, também, como já evidenciado no inquérito nacional de 1980, que a distribuição da DCH na região não é necessariamente linear e homogênea, mas segue padrões de bolsões microregionais, num aspecto de mosaico, especialmente definidos por elementos biológicos, ecológicos, climáticos e sociais (Forattini, 1980; FiusaLima \& Silveira, 1984; Dias \& Coura, 1997).

A sorologia entre crianças escolares mostra, de modo consistente, que nas regiões trabalhadas a transmissão vetorial se encontra virtualmente interrompida, restando alguns raríssimos infectados que podem simplesmente espelhar transmissão congênita. Recentemente, essa hipótese ficou reforçada com um levantamento da FNS/Bahia na região do Recôncavo, que encontrou apenas quatro crianças soropositivas numa amostra de cerca de 12 mil examinadas, sendo aquelas quatro interpretadas como casos congênitos (Vanize O. Macedo, comunicação pessoal). A curva de prevalência por idades de Independência, Ceará (Tabela 14) reflete transmissão mínima, num padrão de médio-baixa endemicidade, característica de regiões endêmicas após controle vetorial, remanescendo indivíduos soropositivos basicamente nas idades adultas (Dias \& Coura, 1997). É relevante que a curva seja comparada também com dados antigos do Ceará, onde
Alencar (1987) detectou em cinco municípios prevalências entre $1,5 \%$ e $2,1 \%$ para escolares e entre $7 \%$ e 19,4\% para indivíduos acima de 29 anos. Nesse importante trabalho, o autor verifica que os maiores índices da infecção humana no nordeste se encontram nas áreas de predomínio de P. megistus e T. infestans, sendo menores nas microregiões onde predomina $T$. brasiliensis e muito menores nas áreas de $T$. pseudomaculata (Alencar, 1987). Isso se confirma razoavelmente pelo cotejamento das prevalências do inquérito nacional da SUCAM com os levantamentos triatomínico-tripanosômicos da mesma instituição no princípio dos anos 80, quando a maior prevalência do nordeste foi na Bahia, com 7,4\% (predomínio de $P$. megistus e T. infestans), seguindo-se Alagoas, com $5,1 \%$ (basicamente $P$. megistus) e Sergipe, com 4,6\% (também P. megistus). Estados com infestação exclusiva por T. brasiliensis e/ou $T$. pseudomaculata mostraram taxas de prevalência significativamente menores, como Ceará $(1,3 \%)$ e Rio Grande do Norte $(1,2 \%)$, aparecendo em graus intermediários Estados com infestação mista (Piauí, Pernambuco e Paraíba), com taxas entre $3,1 \%$ e $2,1 \%$ (Fiusa-Lima \& Silveira, 1984; Silveira et al., 1984a).

As curvas de prevalência por idade também mostram que outras formas de transmissão da DCH idade-dependentes, como a transfusional (maioria dos doadores entre 18 e 50 anos) e a congênita (mulheres férteis entre os 15 e os 40 anos), sob o controle vetorial e com o tempo tendem a diminuir seu impacto (Dias \& Coura, 1997; Dias \& Schofield, 1998).

Nos bancos de sangue nordestinos, um resíduo de infecção chagásica ainda aparece, no seu total, mostrando-se uma prevalência semelhante ou inferior à média brasileira (Tabela 15). A prevalência da DCH nos bancos de sangue tem se mostrado bastante útil através dos tempos e regiões, como indicador da endemicidade chagásica numa região ou período (Schmunis, 1997). É importante assinalar que a hemoterapia no nordeste evoluiu significativamente em qualidade e graus de cobertura, principalmente em alguns Estados, como Pernambuco, onde a proporção de municípios que a praticavam era pouco mais de 52\% em 1989 (MoraesSouza et al., 1994). Dados históricos desse Estado mostravam uma prevalência da infecção chagásica entre 3,7\% e 4,6\% nos anos 70 (Dias, 1979), reduzida para cifras em volta de $0,22 \%$ em 1988 e 0,14\% em 1994, segundo a COSAH Coordenação de Sangue e Hemoderivados/Ministério da Saúde. Esses dados indicam, de um lado, uma tendência à redução da incidência da DCH nas populações mais jovens, mercê do 
controle progressivo dos triatomíneos domiciliados. Por outro, significa também um aperfeiçoamento no sistema hemoterápico e o paulatino afastamento de doadores soropositivos (Dias \& Coura, 1997; Schmunis, 1997).

Como exercício, considerando-se a ocorrência de 350.000 transfusões na região em anos recentes, para uma prevalência da DCH de $0,36 \%$ entre doadores, considerando-se a cobertura mínima de $80 \%$ para sorologia de descarte e o risco máximo de $25 \%$ de transmissão do T. cruzi em sangue infectado e não descartado, poder-se-ia esperar uma ocorrência entre 60 e 80 casos novos/ano (Dias, 1979; Dias \& Schofield, 1998; Schmunis, 1997).

Não há dados ou pesquisa de fôlego sobre a incidência da DCH congênita no nordeste, mas, a se julgar pelo restante do País e pelos dados de sorologia em baixa idade já disponíveis, provavelmente, o seu significado é baixo na região nordestina. Os dados mais consistentes são de Bittencourt (1999), que detectou 0,9\% de transmissão congênita em estudo prospectivo de gestantes chagásicas na Bahia, um dado muito próximo a uma pesquisa entre 18.900 recém nascidos em Minas Gerais, na mesma época (Gontijo et al., 1998). Esses dados são plenamente reforçados pelo estudo referido por Macedo (Vanize O. Macedo, comunicação pessoal) no Recôncavo. Para uma população próxima a 51 milhões de habitantes no Nordeste, em 1999, poder-se-ia esperar a existência de 6.375 .000 mulheres em idade fértil e o nascimento de 1.275 .000 crianças anualmente na região. Aplicando-se o risco de transmissão congênita (0,9\%), calculado por Bittencourt (1999) para um cenário de $2 \%$ e $4 \%$ de gestantes infectadas, resultariam entre 230 e 460 novos casos de DCH pela via congênita anualmente.

No campo da morbi-mortalidade, pode-se também afirmar que a DCH representou e ainda representa impacto significativo no Nordeste brasileiro, estando assinaladas principalmente as formas clínicas da fase crônica, segundo os trabalhos disponíveis. As formas agudas sempre foram raras, segundo Alencar (1987), situação típica de áreas de baixa-média transmissão (Macedo, 1976; Dias \& Coura, 1997). Não obstante, um episódio excepcional ocorreu na $\mathrm{Pa}-$ raíba, em 1988, observando-se um surto de DCH aguda em 26 pessoas, com provável contaminação oral (Shikanai-Yassuda et al., 1991).

Em São Felipe, Bahia, num longo estudo longitudinal e sob observação direta na área, foram diagnosticados apenas 27 casos agudos de transmissão vetorial (Macedo, 1976). De modo geral, entre os pacientes crônicos do Nordeste se descrevem a cardiopatia e as formas digestivas, especialmente a disperistalse esofágica. No Ceará, Alencar (1987) encontrou a cardiopatia em $30,8 \%$ dos soropositivos examinados e alterações digestivas em $11,3 \%$, observando que o gradiente de alterações eletrocardiográficas entre pares de indivíduos chagásicos e não chagásicos era significativamente maior nas áreas de prevalência de P. megistus (26,3\%) que nas de T. brasiliensis (3,3\%). No Recôncavo Baiano, Macedo (1976) detectou 31,3\% de cardiopatia entre 840 chagásicos, com 9,7\% de disperistalse esofágica e $0,7 \%$ de megacólon. Nessa área, muito infestada por P. megistus, detectou-se $56 \%$ dos chagásicos na forma crônica indeterminada entre a população geral. Aparentemente, e com as devidas ressalvas metodológicas, os dados acima indicam maior grau de morbidade na Bahia que no Ceará, o que também irá aparecer na Tabela 16 do presente trabalho, onde a taxa de internação por DCH entre 100.000 habitantes foi de 0,110 para a Bahia e de 0,063 para o Ceará. Entre chagásicos observados no Rio de Janeiro, a prevalência de cardiopatia crônica foi de $66,6 \%$ para 149 casos provenientes da Bahia, com $20,1 \%$ de megas (3/4 de esôfago e 1/4 de cólon), sendo respectivamente $65,7 \%$ e $15,0 \%$ para 53 casos de Pernambuco e $50,9 \%$ e $0,0 \%$ para trinta casos da Paraíba (Coura, 1984). Já em estudos em áreas da Paraíba e do Piauí, o mesmo grupo encontrou um gradiente de global de alterações eletrocardiográficas de $18,3 \%$ entre soropositivos e soronegativos do Piauí e 4,8\% na Paraíba. Esses valores foram significativamente menores que aqueles encontrados pelos mesmos pesquisadores em Minas Gerais, na mesma época. Encontraram também manifestações digestivas nas áreas nordestinas, especialmente a disfagia, com gradientes de $5,8 \%$ na Paraíba e de 5,5\% no Piauí (Coura et al., 1984). Ao longo dos anos parece observar-se, no Nordeste, uma tendência à progressiva diminuição da morbidade da DCH nas áreas em que a densidade tratomínico-tripanosômica vai paulatinamente se reduzindo, mercê do combate aos triatomíneos e a outros fatores como melhoramento social, migrações, etc. Fato como esse foi primeiramente apontado por Emmanuel Dias em Minas Gerais (Dias, 1962) e, posteriormente, reiterado por Macedo (1976), na Bahia, e por Alencar (1987), no Ceará, estando aparentemente de acordo com o que mostra a Tabela 16 do presente trabalho. Ainda em apuração, um inquérito nacional de eletrocardiografia entre pares de indivíduos chagásicos e não chagásicos está em curso pela Universidade de Brasília, podendo aportar importantes conhecimentos sobre a aparente diversidade do da- 
no da esquizotripanose entre estados e regiões do Brasil.

De certa forma, os dados de mortalidade apresentados na Tabela 17 também mostram diversidade no impacto da DCH entre os vários estados nordestinos: mais uma vez, a taxa de mortalidade em virtude da DCH por 100.000 habitantes é maior na Bahia $(4,26)$ que em Pernambuco $(1,68)$ e no Ceará $(0,55)$. Essa diversidade reflete, decerto, a variação entre vetores e cepas locais, intensidade de transmissão e aspectos sociais que explicam os clássicos matizes regionais da DCH (Dias \& Coura, 1997).

O Nordeste como um todo concorreu com $14,27 \%$ dos óbitos por DCH do Brasil em 1997 , apresentando uma taxa por 100.000 habitantes de 1,82, significativamente menor que a do País $(3,38)$. Mais uma vez, reforçam-se as observações de trabalhos anteriores, tanto sobre uma diversidade de impacto da DCH entre os Estados e regiões nordestinas, como a respeito de um menor impacto da doença no Nordeste em relação ao Brasil como um todo e, particularmente, em relação às áreas endêmicas do Sudeste e de Goiás (Coura, 1984; Coura et al., 1984; Alencar, 1997).

\section{Conclusões}

Grosso modo, o perfil esboçado mostra o Nordeste como uma macroregião onde a DCH ocorre endemicamente, com diversidades intra-regionais explicáveis pelas variações ecológicas e sociais, implicando especificamente diferentes vetores e cepas de T. cruzi, intensidade de transmissão e fatores antrópicos. O Nordeste apresenta grande variedade de espécies vetoras que, na prática de saúde pública, definem como importantes basicamente $P$. megistus, $T$. infestans, T. brasiliensis e T. pseudomaculata. As duas primeiras, mais domiciliadas e praticamente sem focos nativos na região, foram eliminadas de vastas áreas nordestinas, princi- palmente mediante o controle químico continuado pela SUCAM/FNS, remanescendo alguns focos residuais que pressupõem prioridade. As duas últimas têm respondido ao programa de controle, mas de modo menos intenso e com muito maior dificuldade operacional, por serem ubiqüistas e nativas na região.

Em todas as áreas trabalhadas houve e tem havido redução de populações triatomínicas domiciliadas, com subseqüente redução nos níveis de transmissão e impacto médico-social da DCH. As demais espécies registradas apresentam pouca ou nenhuma importância epidemiológica e mesmo tendência à primeira domiciliação, com ressalva para $R$. nasutus em algumas sub-regiões. O Maranhão é o estado com menor impacto da doença, apresentando baixa infestação e ausência dos triatomíneos causadores das maiores taxas de transmissão e morbidade (T. infestans, P. megistus). Ao contrário, Bahia, Alagoas, Sergipe, Pernambuco e Piauí mostram os índices de doença mais significativos. No todo, a DCH ainda é importante no Nordeste, embora seus índices de morbimortalidade aparentemente sejam menores que os do Sudeste e os de Goiás. Não obstante, o Nordeste é hoje a região do Brasil que mais deve preocupar as autoridades sanitárias, justamente pela remanescência de espécies nativas com potencial invasivo e de difícil controle como T. brasiliensis e T. pseudomaculata. Acresce ainda estar nessa região (Bahia) um dos dois últimos grandes focos de T. infestans, que tanto pode ser eliminado como servir de ponta de lança para uma re-expansão da espécie.

Finalmente, é preocupante a visível deterioração da FNS na região, por determinantes políticos e administrativos, com nítida diminuição de força de trabalho e desativação de extensas áreas de vigilância epidemiológica, sem a devida assimilação de suas atividades pelos estados e municípios, como esperável no processo de descentralização.

\section{Agradecimentos}

Os autores expressam seus agradecimentos às gerências técnicas de doença de Chagas da Fundação Nacional de Saúde do Maranhão, Ceará, Piauí, Rio Grande do Norte, Paraíba, Pernambuco, Alagoas, Sergipe e Bahia pelos aportes e esclarecimentos apresentados tanto na reunião de 1998 (Praia das Fontes/Ceará) como nos contatos seguintes. Agradecem também aos Drs. Hélio Morais Souza (Coordenação Nacional de Sangue e Hemoderivados, Mato Grosso do Sul), Vanise Macedo (Universidade de Brasília) e J. Rodrigues Coura (Instituto Oswaldo Cruz, Fundação Oswaldo Cruz), pelas sugestões e aportes ao texto. 


\section{Referências}

A IMPRENSA, 1911. Entrevista com Carlos Chagas. $A$ Imprensa, Rio de Janeiro, 6 ago., p. 4.

ALENCAR, J. E., 1987. História Natural da Doença de Chagas no Estado do Ceará. Fortaleza: Imprensa Universitária, Universidade Federal do Ceará.

BITTENCOURT, A. L., 1999. Transmissão vertical da doença de Chagas. In: Trypanosoma cruzi $e$ Doença de Chagas (Z. Brener, Z. Andrade \& M. Barral-Netto, org.), pp. 16-20, Rio de Janeiro: Guanabara Koogan.

CARCAVALLO, R. U. \& TONN, R. J., 1985. Rhodnius prolixus Stal. In: Factores Biológicos y Ecológicos en la Enfermedad de Chagas (R. U. Carcavallo, J. E. Rabinovich \& R. J. Tonn, org.), pp. 209-217, Buenos Aires: Servicio Nacional de Chagas.

CARCAVALLO, R. U.; GIRON, I. G.; JURBERG, J. \& LENT, H., 1997. Atlas dos Vetores da Doença de Chagas nas Américas. Rio de Janeiro: Editora Fiocruz.

CASTRO FILHO, J. \& SILVEIRA, A. C., 1984. Distribuição da doença de Chagas no Brasil. Revista Brasileira de Malariologia e Doenças Tropicais, 31:8597.

CHAGAS, C. R. J., 1916. Tripanosomíase Americana: Forma aguda da moléstia. Memórias do Instituto Oswaldo Cruz, 8:37-69.

CHAGAS, C. R. J., 1930. Forma cardíaca da Tripanosomiase americana. Memórias do Instituto Oswaldo Cruz, 24:89-109.

COSTA, J., 1999. The synanthropic process of Chagas Disease vectors in Brazil, with special attention to Triatoma brasiliensis Neiva, 1911 (Hemiptera, Reduviidae, Triatominae). Population, genetical, ecological and epidemiological aspects. Memórias do Instituto Oswaldo Cruz, 94:230-239.

COURA, J. R., 1984. Doença de Chagas como endemia urbana. In: Cardiopatia Chagásica (J. R. Cançado \& M. Chuster, org.), pp. 356-361, Belo Horizonte: Fundação Carlos Chagas.

COURA, J. R.; ABREU, L. L.; DUBOIS, L. E. G.; LIMA, F. C.; ARRUDA Jr., E. R.; WILLCOX, H. P. F.; ANUNZIATTO, N. \& PETANA, W., 1984. Morbidade da doença de Chagas. II - Estudos seccionais em quatro áreas de campo no Brasil. Memórias do Instituto Oswaldo Cruz, 79:101-124.

DATASUS (Departamento de Informática do SUS), 1999. Mortalidade geral no Brasil. <http:// www. datasus.gov.br>.

DIAS, E., 1936. Xenodiagnóstico e algumas verificações epidemiológicas na doença de Chagas. $\mathrm{Mi}$ sión de Patología Regional, 1:89-119.

DIAS, E., 1962. Os efeitos da superinfecção sobre a evolução da cardiopatia crônica chagásica. Revista Goiana de Medicina, 9:233-242.

DIAS, E.; LARANJA, F. S. \& NÓBREGA, G. C. C., 1945. Doença de Chagas. Memórias do Instituto Oswaldo Cruz, 43:495-582.

DIAS, J. C. P., 1979. Mecanismos de transmissão. In: Trypanosoma cruzi e Doença de Chagas (Z. Brener \& Z. A. Andrade, org.), pp. 152-174, Rio de Janeiro: Guanabara Koogan.

DIAS, J. C. P., 1991. Chagas Disease control in Brazil: Which strategy after the attack phase? Annales de la Societé Belge de Medicine Tropicale, 71:7586.
DIAS, J. C. P., 1998. Problemas e possibilidades de participação comunitária no controle das grandes endemias no Brasil. Cadernos de Saúde Pública, 14:19-37.

DIAS, J. C. P. \& COURA, J. R., 1997. Epidemiologia. In: Clínica e Terapêutica da Doença de Chagas. Um Manual Prático para o Clínico Geral (J. C. P. Dias \& J. R. Coura, org.), pp. 33-66, Rio de Janeiro: Editora Fiocruz.

DIAS, J. C. P. \& SCHOFIELD, C. J., 1998. Controle da transmissão transfusional da doença de Chagas na Iniciativa do Cone Sul. Revista da Sociedade Brasileira de Medicina Tropical, 31:373-383.

DIOTAIUTI, L.; LOIOLA, C. F; FALCÃO, P. L. \& DIAS, J. C. P., 1993. The ecology of Triatoma sordida in natural environments in two different regions of the state of Minas Gerais, Brazil. Revista do Instituto de Medicina Tropical de São Paulo, 35:237-245.

DIOTAIUTI, L.; SILVEIRA, A. C. \& ELIAS, M., 1984. Encontro de $R$. prolixus Stal, 1859, em Macaubeiras. Revista Brasileira de Malariologia e Doenças Tropicais, 36:11-14.

DIOTAIUTI, L.; FARIA FILHO, O. F.; CARNEIRO, F. F.; ROMANHA, A. J.; PIRES, H. H. R.; BORGES, E. C. \& SCHOFIELD, C. J., 1998. Potencial vetorial e controle do Triatoma brasiliensis e do Triatoma pseudomaculata no nordeste do Brasil. In: Proceedings of The $3^{\text {rd }}$ International Workshop on Population Genetics and Control of Triatominae (L. Diotaiuti \& C. J. Schofield, org.), pp. 37-43, Belo Horizonte: Centro de Pesquisas René Rachou, Fundação Oswaldo Cruz.

DUJARDIN, J. P.; PANZERA, F. \& SCHOFIELD, C. J., 1999. Triatominae as a model to morphological plasticity under ecological pressure. Memórias do Instituto Oswaldo Cruz, 94:223-228.

FIUSA-LIMA, J. T. \& SILVEIRA, A. C., 1984. Controle da transmissão e inquérito sorológico nacional. In: Cardiopatia Chagásica (J. R. Cançado \& M. Chuster, org.), pp. 371-380, Belo Horizonte: Fundação Carlos Chagas.

FORATTINI, O. P., 1980. Biogeografia, origem e distribuição da domiciliação de triatomíneos no Brasil. Revista de Saúde Pública, 14:285-299.

GONTIJO, E. D.; JANUZZI, J. H.; MOREIRA, E.; JANUÁRIO, N.; MOURÃO, O.; TAVARES, V.; OLIVEIRA, C. M.; LAMEGO, A. C. \& DIAS, J. C. P., 1998. Doença de Chagas congênita - Inquérito sorológico em Minas Gerais - Modelo e proposta. Revista da Sociedade Brasileira de Medicina Tropical, 31:53-54.

IBGE (Fundação Instituto Brasileiro de Geografia e Estatística), 1991. Censo Demográfico 1991. Resultados do Universo Relativos às Características da População e dos Domicílios. Rio de Janeiro: Secretaria de Planejamento, Orçamento e Coordenacão, IBGE.

IBGE (Fundação Instituto Brasileiro de Geografia e Estatística), 1999. <http://ibge.gov.br>.

LENT, H. \& WIGODZINSKY, P., 1979. Revision of the Triatominae (Hemiptera, Reduviidae), and their significance as vectors of Chagas' disease. Bulletin of the American Museum of Natural History, 163:125-520. 
LUCENA, D. T., 1952. Subsídios para o estudo epidemiológico da doença de Chagas no nordeste. Revista Brasileira de Malariologia e Doenças Tropicais, 4:171-175.

LUTZ, A. \& MACHADO, A., 1915. Viagem pelo Rio São Francisco e por alguns dos afluentes entre Pirapora e Juazeiro. Memórias do Instituto Oswaldo Cruz, 7:5-62.

MACEDO, V. O., 1976. Influência da exposição à reinfecção na evolução da doença de Chagas (estudo longitudinal de cinco anos). Revista de Patologia Tropical, 5:33-116.

MORAES-SOUZA, H.; WANDERLEY, D. M. V.; BRENER, S.; NASCIMENTO, R. D.; ANTUNES, C. M. F \& DIAS, J. C. P., 1994. Hemoterapia e doença de Chagas transfusional no Brasil. Boletín de la Oficina Sanitaria Panamericana, 116:406-418.

NEIVA, A., 1911. Contribuição para o estudo dos hematófagos brasileiros e descrição de uma nova espécie de Triatoma. Brasil Médico, 25:461-462.

NEIVA, A., 1944. Triatomídeos. Coletânea IV. Rio de Janeiro: Imprensa Oficial.

NEIVA, A. \& LENT, H., 1941. Sinopse dos triatomídeos. Revista de Entomologia, 12:61-92.

NEIVA, A. \& PENA, B., 1916. Viagem científica pelo norte da Bahia, sul do Piauí e de norte a sul de Goiás. Memórias do Instituto Oswaldo Cruz, 8:74224.

PINTO, C., 1925. Ensaio Monographico dos Eduvideos Hematophagos ou "Barbeiros". Tese de Doutorado, Rio de Janeiro: Faculdade de Medicina, Universidade do Rio de Janeiro.

PIRAJÁ-DA-SILVA, M. A., 1913. O barbeiro (Conorhinus megistus Burm.) na Bahia. Arquivos Brasileiros de Medicina, 1:627-632.

REZENDE, J. M., 1998. Megaesôfago chagásico. Dados históricos sobre o reconhecimento de sua etiologia. Gastroenterologia Contemporânea, 2:6-15.

ROCHA-E-SILVA, E. O.; WANDERLEY, D. M. V. \& RODRIGUES, V. L. C. C., 1998. Triatoma infestans: Sua importância, controle e eliminação da espécie no Estado de São Paulo, Brasil. Revista da Sociedade Brasileira de Medicina Tropical, 31:73-88.
SCHMUNIS, G. A., 1997. Tripanossomíase americana: Seu impacto nas Américas e perspectivas de eliminação. In: Clínica e Terapêutica da Doença de Chagas. Um Manual Prático para o Clínico Geral (J. C. P. Dias \& J. R. Coura, org.), pp. 11-24, Rio de Janeiro: Editora Fiocruz.

SCHOFIELD, C. J., 1994. Triatominae: Biología y Control. London: Eurocommunica Publications.

SCHOFIELD, C. J. \& DIAS, J. C. P., 1998. The Southern Cone initiative against Chagas disease. Advances in Parasitology, 42:2-27.

SHIKANAI-YASSUDA, M. A.; MARCONDES, C. B.; GUEDES, L. A.; SIQUEIRA, G. S.; BARONE, A. A.; DIAS, J. C. P.; AMATO NETO, V.; TOLEZANO, J. E.; PERES, B. A.; ARRUDA Jr., E. R.; LOPES, M. H.; SHIROMA, M. \& CHAPADEIRO, E., 1991. Possible oral transmission of acute Chagas' disease in Brazil. Revista do Instituto de Medicina Tropical de São Paulo, 33:351-357.

SILVEIRA, A. C. \& REZENDE, D. F., 1994. Epidemiologia e controle da transmissão vetorial da doença de Chagas no Brasil. Revista da Sociedade Brasileira de Medicina Tropical, 27 (Sup. 3):11-22.

SILVEIRA, A. C. \& VINHAES, M., 1998. Doença de Chagas: Aspectos epidemiológicos e de controle. Revista da Sociedade Brasileira de Medicina Tropical, 31:15-60.

SILVEIRA, A. C.; FEITOSA, V. R. \& BORGES, R., 1984a. Distribuição de triatomíneos domiciliados no período 1975/1983 no Brasil. Revista Brasileira de Malariologia e Doenças Tropicais, 36:15-312.

SILVEIRA, A. C.; REZENDE, D. F. \& MÁXIMO, M. H. C., 1984b. Risk measure of domestic transmission of Chagas' Disease, through a new entomological indicator. Memórias do Instituto Oswaldo Cruz, 79:113-116.

SUCAM (Superintendência de Campanhas de Saúde Pública), 1980. Manual de Normas Técnicas da Campanha de Controle da Doença de Chagas. Brasília: Ministério da Saúde.

WHO (World Health Organization), 1991. Control of Chagas Disease. WHO Technical Report Series 811. Geneva: WHO. 